PAPER

\section{Triple shape memory polymers by 4D printing}

To cite this article: M Bodaghi et al 2018 Smart Mater. Struct. 27065010

View the article online for updates and enhancements.
Related content

Increasing dimension of structures by 4D printing shape memory polymers via fused deposition modeling

G F Hu, A R Damanpack, M Bodaghi et al.

Self-Expanding/Shrinking Structures by 4D Printing

M Bodaghi, A R Damanpack and W H Liao

Thermo-mechanical modeling of semi-

crystalline thermoplastic shape memory

polymer under large strain

R Bouaziz, F Roger and K Prashantha 


\title{
Triple shape memory polymers by 4D printing
}

\section{Bodaghi, A R Damanpack and W H Liao}

Smart Materials and Structures Laboratory, Department of Mechanical and Automation Engineering, The Chinese University of Hong Kong, Shatin, N.T., Hong Kong, People's Republic of China

E-mail:whliao@cuhk.edu.hk

Received 30 January 2018, revised 14 March 2018

Accepted for publication 6 April 2018

Published 8 May 2018

\begin{abstract}
This article aims at introducing triple shape memory polymers (SMPs) by four-dimensional (4D) printing technology and shaping adaptive structures for mechanical/bio-medical devices. The main approach is based on arranging hot-cold programming of SMPs with fused decomposition modeling technology to engineer adaptive structures with triple shape memory effect (SME). Experiments are conducted to characterize elasto-plastic and hyper-elastic thermo-mechanical material properties of SMPs in low and high temperatures at large deformation regime. The feasibility of the dual and triple SMPs with self-bending features is demonstrated experimentally. It is advantageous in situations either where it is desired to perform mechanical manipulations on the 4D printed objects for specific purposes or when they experience cold programming inevitably before activation. A phenomenological 3D constitutive model is developed for quantitative understanding of dual/triple SME of SMPs fabricated by 4D printing in the large deformation range. Governing equations of equilibrium are established for adaptive structures on the basis of the nonlinear Green-Lagrange strains. They are then solved by developing a finite element approach along with an elastic-predictor plastic-corrector return map procedure accomplished by the Newton-Raphson method. The computational tool is applied to simulate dual/triple SMP structures enabled by 4D printing and explore hot-cold programming mechanisms behind material tailoring. It is shown that the 4D printed dual/triple SMPs have great potential in mechanical/bio-medical applications such as self-bending gripers/stents and self-shrinking/tightening staples.
\end{abstract}

Keywords: 4D printing, hot-cold programming, dual/triple SMPs, constitutive modeling, experiments, FEM

(Some figures may appear in colour only in the online journal)

\section{Introduction}

Shape memory polymers (SMPs) are a class of multi-functional smart materials that are capable of being deformed to temporary shapes and of recovering to their original shape in response to external stimuli, such as temperature [1]. Beyond the unique shape memory effect (SME), SMPs have many advantages including light weight, low cost, highly flexible programming, good biocompatibility and biodegradability. These superior properties have made SMPs attractive for use in many engineering applications such bio-medical devices, deployable space structures, shape-morphing structures and micro-electro-mechanical systems [2, 3]. World-wide scientific efforts have been devoted to describe thermomechanical behaviors of SMPs in predictive modeling frames. A comprehensive review of SMP constitutive models can be found in works by Liu et al [4] and Park et al [5].

Most SMPs can only memorize one shape, called the dual SME. The generic shape memory cycle in thermallyinduced dual SMPs mostly consists of a shape programming protocol followed by a shape recovery driven by heating. The shape programming can be classified as hot and cold, on the basis of the temperature zone in which the programming is performed. A schematic of the hot and cold programming for dual SME is shown in figures 1 and 2, respectively, in which $\sigma, \varepsilon$ and $T$ stand for stress, strain and temperature. In the case 


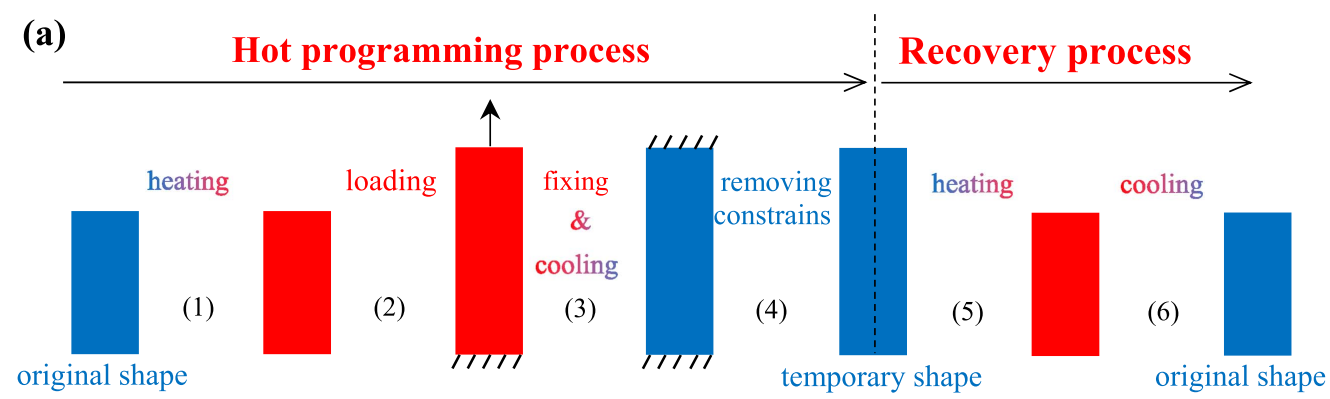

(b)

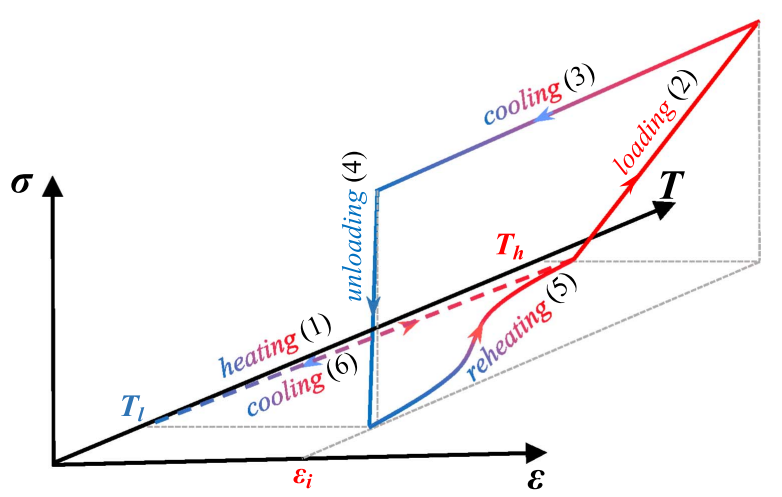

Figure 1. Hot programming for dual SME: (a) scheme, (b) stress-strain-temperature diagram.

(a)

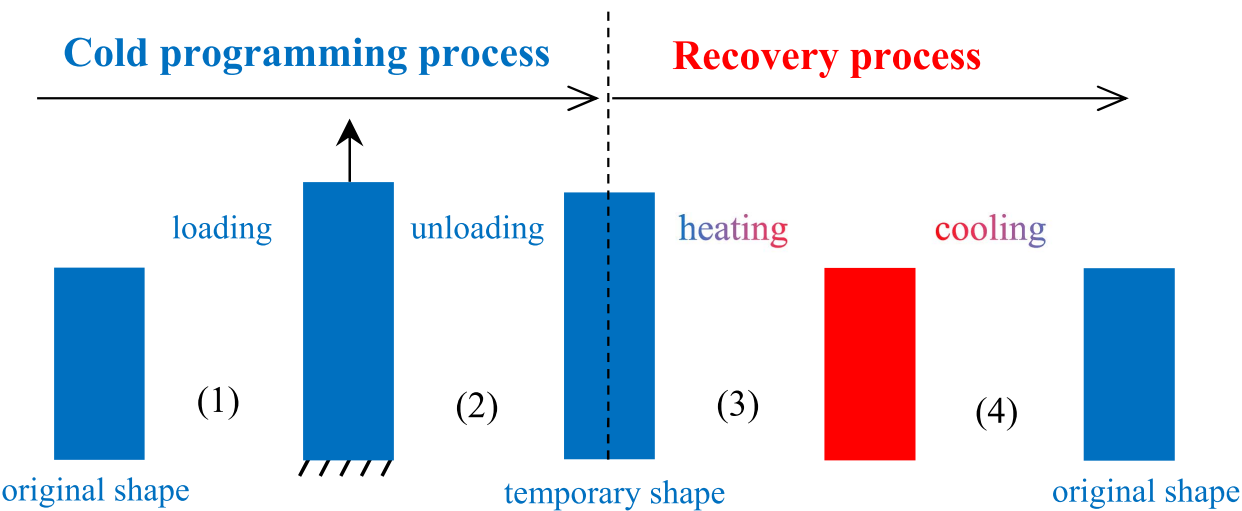

(b)

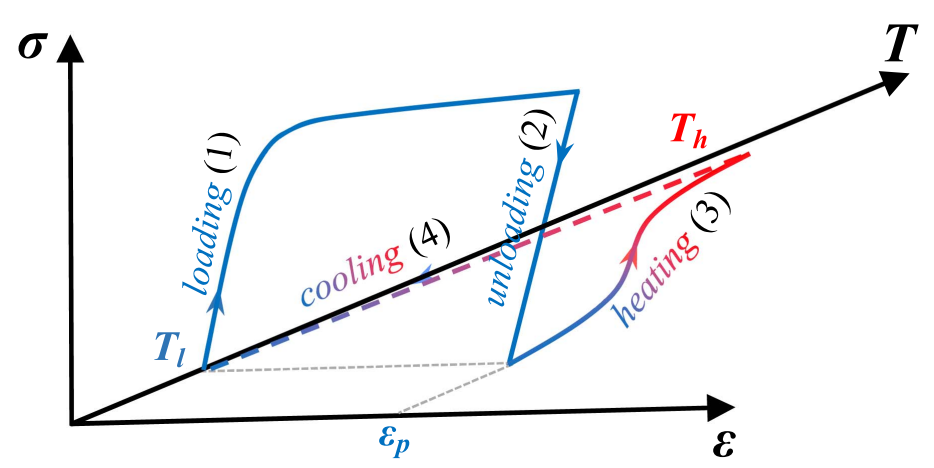

Figure 2. Cold programming for dual SME: (a) scheme, (b) stress-strain-temperature diagram.

of hot programming for dual SME as shown in figure 1, the material initially in a strain/stress-free state at a temperature lower than glassy transition temperature $\left(T=T_{l}<T_{g}\right)$ is heated above the transition temperature range $\left(T=T_{h}>T_{g}\right)$ (step 1). The material stable at rubbery phase is then loaded mechanically and cooled down while being kept fixed memorizing a temporary shape (steps 2 and 3 ). The SMP is unloaded by releasing the constraint while a inelastic stain, 
called pre-strain, $\varepsilon_{i}$, remains in the material (step 4). Next, the SMP is heated to recover to its original shape known as free strain recovery (steps 5), see figure 1. Finally, it is cooled back to low temperature (step 6). In the case of cold programming for dual SME, the material initially in a strain/ stress-free glassy state at a low temperature is first deformed mechanically, then unloaded to a temporary shape, and finally heated up to recover the original shape, see figure 2 . The mechanical load must be large enough to induce the reversible plastic strain, $\varepsilon_{p}$, into the material. The hot programming advantage is that the polymer can easily be deformed into a desired large temporary shape with a small force and very small spring-back [6]. However, it has some limitations like the heating process that is a time and energy consuming procedure. In this case, cold programming becomes attractive in terms of saving time and energy specially many load-carry SMPs are stable in their glassy phase at room temperature.

Recent advances in additive manufacturing has introduced four-dimensional (4D) printing technology for fabricating smart architectures and metamaterials that can be switched between multiple configurations via environmental stimuli like heat and/or humidity [7-15]. For example, Mao et al [12] introduced shape-changing components by 3D printing curable liquid photo-polymers via PolyJet ${ }^{\mathrm{TM}}$ technology. They printed multi-material composite structures where hydrogel was confined by SMP and elastomer layers. The printed structures self-folded by dipping in low, then high temperature water, cooling down, drying and finally unfolded upon putting in high temperature water. Ding et al [13] presented self-folding composite architectures consisting of SMP and elastomer with built-in compressive strains induced during photo-polymerization of PolyJet process. López-Valdeolivas et al [14] designed soft self-foldable actuators by ink 3D printing cross-linked liquid crystalline polymers fixed through photo-polymerization. Bodaghi et al [15] programmed metamaterials with dual SME by fused decomposition modeling (FDM) printing technology.

Unlike conventional dual SMPs that can recover from a temporary shape to an original shape, triple SMPs have potentials of memorizing two temporary shapes and sequentially recovering their original shapes from two temporary shapes by simply heating [16-18]. Two methods are usually employed to make SMPs that exhibit triple SME [19]. The first approach is the incorporation of two SMPs with various transition temperatures into polymer networks. The second one is utilizing an SMP with a broad transition temperature. Compared to dual SMPs, triple SMPs can meet more complex requirements of applications with a demand of continuous shape change [19].

Although some fabrication approaches have been developed for directly printing dual SMPs, printable capability of triple SMPs has been left as a challenge. This work aims at introducing 4D printed triple SMPs by a combination of hot and cold programming processes. The SMP is first programmed during printing by filament-based FDM technology to get its first temporary shape. It then experiences a cold programming process to memorize the second temporary shape. Finally, it recovers to its original shape in a step-by- step manner from two pre-programmed temporary shapes. This approach is advantageous in situations either where it is desired to perform mechanical manipulations on the 4D printed objects for specific purposes or when they experience cold programming inevitably before activation. Often the latter may happen during installing, storing and packing of the printed objects. For example, bio-medical stents need to be stored inside a catheter for delivery to an artery. In the rest of the paper, after description of the concept behind 4D printed triple SMPs, its feasibility and performance are assessed experimentally. It is also revealed that the material behaves elasto-plastically and hyper-elastically in low and high temperatures at large deformation regime. In order to enable design dual/triple SMP structures by 4D printing, a 3D phenomenological constitutive model is developed to incorporate elasto-plastic and hyper-elastic material behaviors in the finite strain regime during hot-cold programming and activation phase. It is then implemented into a finite element (FE) formulation to simulate 4D printed triple SMP components through an elastic-predictor plastic-corrector return map approach. It is shown experimentally and numerically that the 4D printed dual/triple SMPs can serve as self-bending/ shrinking gripers, stents and staples for mechanical and biomedical applications. Due to the absence of similar concept, mathematical model and results in the specialized literature, this paper is likely to advance the state of the art triple SMPs by $4 \mathrm{D}$ printing technology.

\section{Materials and design}

\subsection{Conceptual design}

The main idea is based on arranging hot-cold programming of SMPs with FDM printing technology to engineer adaptive structures with triple plasticity-based SME. The conceptual approach is illustrated in figure 3, where the SMP is printed and programmed along with a step-by-step thermo-mechanical protocol.

First, FDM as a filament-based material-extrusion 3D printing technology is implemented to fabricate and program SMPs for dual SME at the same time. The SMP filament is supplied from a reel and fed into a heated liquefier where it is molten at temperature $T_{h}$ that is higher than its $T_{g}$. The molten material is forced out of the circular nozzle of the printer head and deposited on the build tray or exciting layers. The extrusion head is moved by servo motors at speed $V_{p}$ following a toolpath controlled by a computer-aided manufacturing software package to define the printed shape. This movement may stretch the molten SMP fiber that is similar to loading stage in the hot programming process, see figures 1 and 3. The pre-strain induced into the SMP during hot programming is directly related to mechanical loading provided by moving printer head. Thus, any increase in printing speed may affect the pre-strain value. The printed fiber cools and solidifies quickly upon contact with build tray and/or layers deposited earlier. This is similar to the step 3 in hot programming process as shown in figure 1 . Once a layer is 


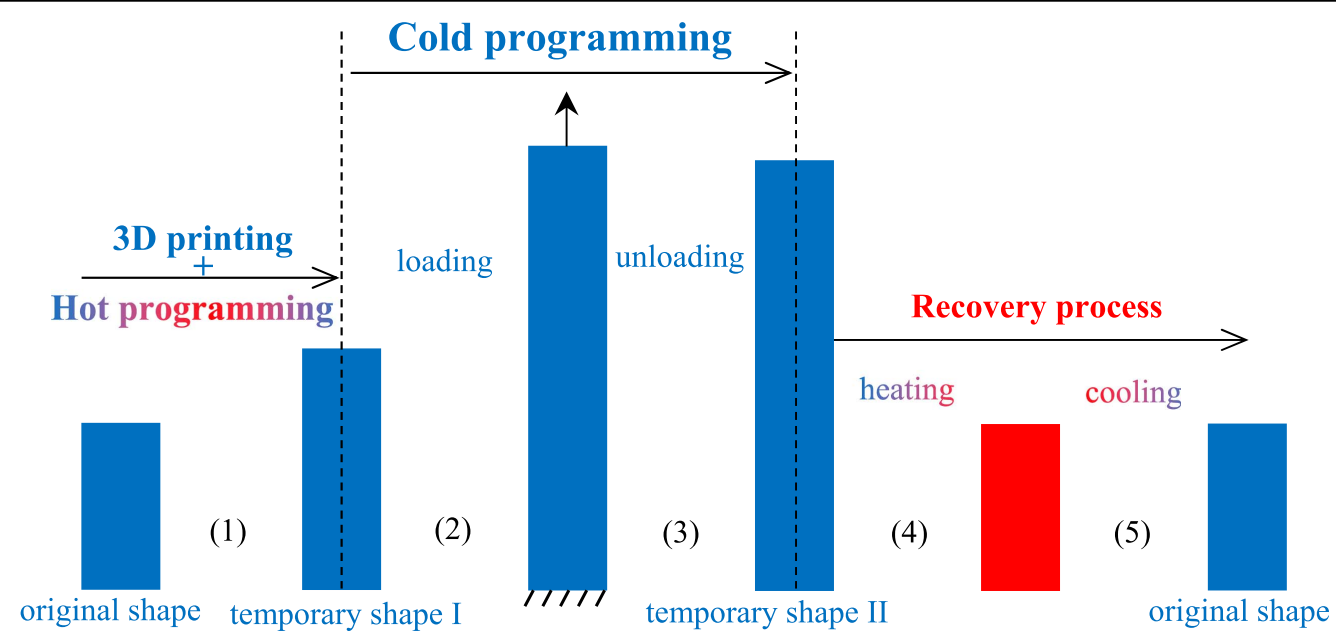

Figure 3. Schematic of 4D printing combined with hot-cold programming for triple SME.

printed, the platform is lowered down to begin the next layer. The printing procedure continues until the fabrication of the object is completed. During this layer-by-layer fabrication, every layer may experience different thermal/bonding conditions at its top and bottom surfaces. For instance, while the first layer is printed on the stiff tray with a prescribed temperature, the rest layers are deposited on the already printed SMP layers which are cooling and solidifying. Therefore, stiffness, temperature and surface condition of the tray may affect bonding, constraint and cooling conditions and consequently the pre-strain regime. Moreover, all layers except the final layer are heated locally at their top surface by the molten SMP when the printer is depositing the upper layer. Thus, temperature of the liquefier and the layer that is being heated play important roles in determining the pre-strain regime. In this respect, the surface heating by the molten SMP fiber may reduce the pre-strain value of the layer beneath it. As the end printed layer is never heated, its pre-strain may be higher than other layers. Furthermore, the temperature of the layer being heated is directly associated with the delay time between printing each layer. The hot programming process is finally completed by removing the printed object from the platform that is like step 5 in figure 1 . The printed configuration can be considered as the first temporary shape of the SMP architecture with built-in pre-strain. Since six printing factors, namely stiffness/surface condition/temperature of build tray, liquefier-temperature, printing speed and time-delay may have potentials to induce non-uniform hot programming process, the pre-strain regime may be anisotropic toward the building direction.

The printed object is expected to have potential to transform into a second temporary shape by cold programming process, see figures 2 and 3. As most of SMPs are stable in their glassy phase state at room temperature, cold programming may imply programming at room temperature that would be attractive in terms of saving time and energy. Cold programming includes a mechanical loading-unloading so as to result in a residual plastic strain and consequently the second temporary shape. When the 4D printed is subjected to an applied stress that exceeds a yield stress level, the plasticity process is initiated, see figure 2(b). The material softens and experiences a plateau in the stress-strain diagram. The material is then unloaded elastically while plastic deformations remain into it. The $4 \mathrm{D}$ printed structure may involve both inelastic built-in and plastic strains induced by hot and cold programming processes, respectively. It could have the capability to recover to its original shape step-by-step from two programmed temporary shapes upon heating revealing triple plasticity-based SME.

\subsection{Materials and fabrication}

The SMP material used for 3D printing is polyurethane-based filaments with a $1.75 \mathrm{~mm}$ diameter and glass transition temperature of $55^{\circ} \mathrm{C}$ manufactured and supplied by SMP Technologies Inc., Tokyo, Japan. The objects are printed using a NewCreator Pro 3D printer manufactured by FlashForge. It is based on the FDM printing technology and equipped by an extrusion nozzle with a physical diameter of $0.4 \mathrm{~mm}$. CraftWare software is used to convert digital 3D object drawn by SolidWorks into G-code toolpath format. Printrun software is also utilized to handle the $3 \mathrm{D}$ printing process. In all printing in this study, unless otherwise stated, the temperature of the liquefier, build tray and chamber is adjusted to $230{ }^{\circ} \mathrm{C}, 23{ }^{\circ} \mathrm{C}$ and $23{ }^{\circ} \mathrm{C}$, respectively, and the printing speed is set at $10 \mathrm{~mm} \mathrm{~s}^{-1}$. Each printing layer is considered to have $0.2 \mathrm{~mm}$ thickness with filling density of $100 \%$. The raster angle is fixed to be $0^{\circ}$. It means all objects are filled in such way that the printing raster is along the longest direction especially parallel to the length of the gauge section of measuring samples. It should be mentioned that the layer-by-layer 3D printing may introduce material anisotropy into the printed objects. Although material properties presented in this section can be considered as material properties along with the printing direction, anisotropy effects are ignored in this study. It will be revealed numerically that assuming isotropic material properties is reasonable for the 3D objects printed in this research.

Elastic thermo-mechanical properties of the printed SMP are measured via a dynamic-mechanical analyzer (DMA) 


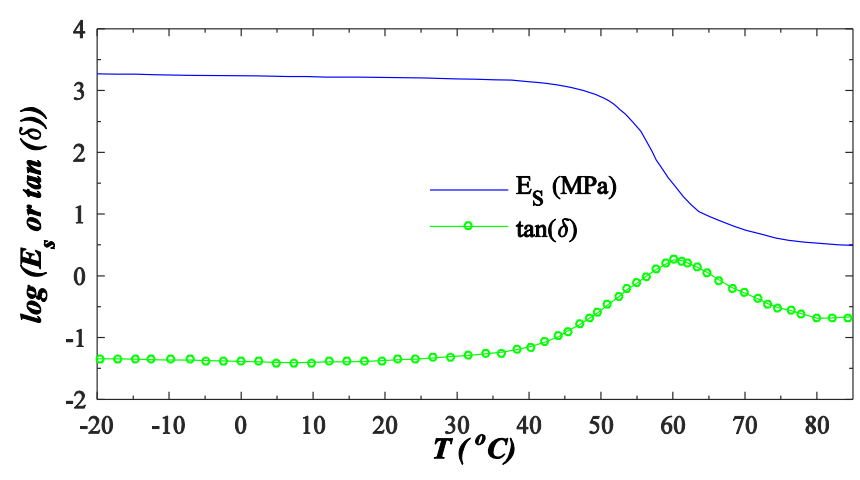

Figure 4. DMA test of the printed SMP: storage modulus and $\tan (\delta)$ versus temperature.

(Model 242, NETZSCH) in tension. DMA samples with dimension of $16 \times 1.6 \times 1 \mathrm{~mm}$ (length-width-thickness) are printed. The test is performed in the axial mode along the printing direction with frequency of force oscillation $1 \mathrm{~Hz}$ and heating ramp of $5{ }^{\circ} \mathrm{C} \min ^{-1}$ varying from $-20{ }^{\circ} \mathrm{C}$ to $85^{\circ} \mathrm{C}$. Ratio of dynamic stress to static stress is about 1.5 . Figure 4 displays the thermo-mechanical behavior of the printed SMP in terms of storage modulus, $E_{S}$, and phase lag, $\tan (\delta)$. It is seen that the storage modulus of the printed SMP in the glassy phase at $T_{l}=23{ }^{\circ} \mathrm{C}$ read $1.66 \mathrm{GPa}$ reduces significantly 522 times to $3.18 \mathrm{MPa}$ at rubbery phase in the temperature of $T_{h}=85^{\circ} \mathrm{C}$. It is also observed that the phase lag peaks at $60{ }^{\circ} \mathrm{C}$ that is considered as the glass transition temperature, $T_{g}=60^{\circ} \mathrm{C}$.

Next, tension and thermal strain tests are conducted to explore elastic thermo-mechanical responses of the printed SMP at low/high temperatures in terms of Young's modulus $(E)$, Poisson's ratio $(\nu)$ and thermal expansion coefficient $(\alpha)$. Samples are printed based on the geometry and dimensions arranged by the ASTM D638 standard [20]. Uniaxial tensile tests are carried out using Tinius Olsen ${ }^{\circledR} H 5 k 5$ (Horsham, PA, USA) equipped with a $5 \mathrm{kN}$ load-cell and a home-made thermal chamber. Material tests are performed at $23{ }^{\circ} \mathrm{C}$ and $85^{\circ} \mathrm{C}$ when the material is stable at glassy and rubbery phases. All tests are conducted at a constant speed of $1 \mathrm{~mm} \mathrm{~min}{ }^{-1}$. Young's modulus defined as slope of stressstrain diagram in the small strain range are measured as $E_{g}=1.66 \mathrm{GPa}$ and $E_{r}=3.3 \mathrm{MPa}$. Poisson's ratio and thermal expansion coefficient are also measured as $v_{g}=0.35, \alpha_{g}=10^{-4} \mathrm{~K}^{-1}$ and $v_{r}=0.44, \alpha_{r}=10^{-4} \mathrm{~K}^{-1}$. Subscripts ' $g$ ' and ' $r$ ' stand for the glassy and rubbery phases, respectively, here and henceforth. It can be found that, while thermal expansion coefficient has no significant change during glassy-to-rubbery phase transformation, Poisson's ratio increases $26 \%$ to the value of 0.44 . The present SMP is supposed to become incompressible at its rubbery phase due to the high rubbery phase Poisson's ratio.

In many types of applications, SMPs may experience large rotations and moderate/finite strains. Therefore, it is necessary to examine behavior of the printed SMP in the large-strain range. To this end, tensile samples at temperatures of $23{ }^{\circ} \mathrm{C}$ and $85^{\circ} \mathrm{C}$ are stretched up to $50 \%$ strain and then unloaded. The results in terms of engineering stress-strain, (a)

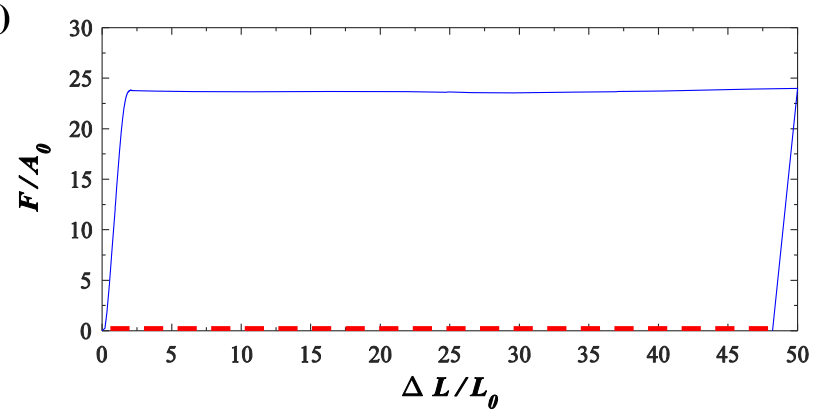

(b)

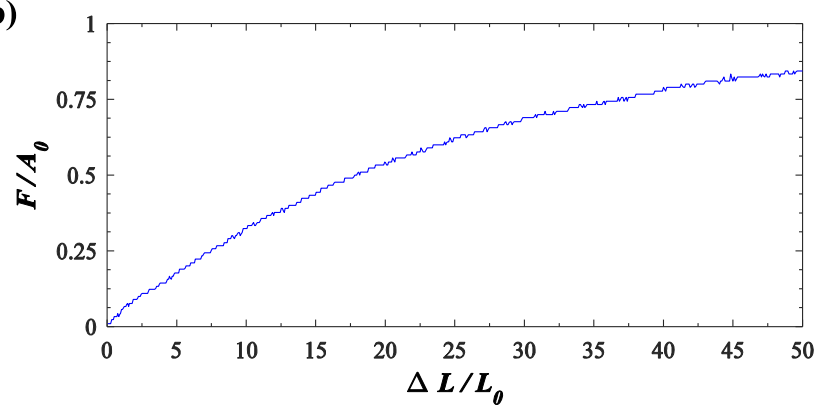

Figure 5. Uniaxial tensile test of the printed SMP at low and high temperatures: (a) $23{ }^{\circ} \mathrm{C}$, (b) $85^{\circ} \mathrm{C}$. (for $T=23{ }^{\circ} \mathrm{C}$ strain recovery induced by the temperature rise is indicated with a red dashed line)

$F / A_{0}-\Delta L / L_{0}$, are illustrated in figures 5(a) and (b) for low and high temperatures, respectively. $F$ and $\Delta L$ stand for force and displacement while $A_{0}$ and $L_{0}$ are initial cross-sectional area and length, respectively. The results presented in figure 5 reveals that the printed SMP behaves elastically in a linear manner within a small strain range of $2 \%$ and $5 \%$ at low and high temperatures. Beyond these strains, the SMP shows a softening response with different deformation mechanism depending on the temperature. The SMP stable in the glassy phase at low temperature behaves elasto-plastically, see figure 5(a). It experiences a nearly flat plateau during plastic deformation. When the force is released gradually by unloading, the SMP unloads elastically while a plastic strain of $48 \%$ remains into it at the end of unloading. However, this plastic stain can be recovered by heating SMP to the temperature of $85^{\circ} \mathrm{C}$ as presented by a dashed line in figure 5(a). It implies the mechanically induced plastic strain is fully recoverable. Thus, it can be found that the printed SMP has potential for plasticity-based cold programming and its applications. At high temperatures, although engineering strain is moderately large, $50 \%$, the printed SMP stable in the rubbery phase behaves elastically in a nonlinear manner, see figure 5(b). The SMP at high temperatures acts like incompressible rubber-like hyper-elastic materials [21]. It can finally be concluded from figure 5 that the SMP behavior from elasto-plastic at low temperatures changes to hyperelastic at higher temperatures.

\subsection{Feasibility study}

In this section, feasibility and performance of the dual/triple SMP by $4 \mathrm{D}$ printing are verified experimentally. First, 


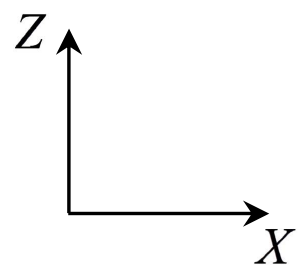

(a)
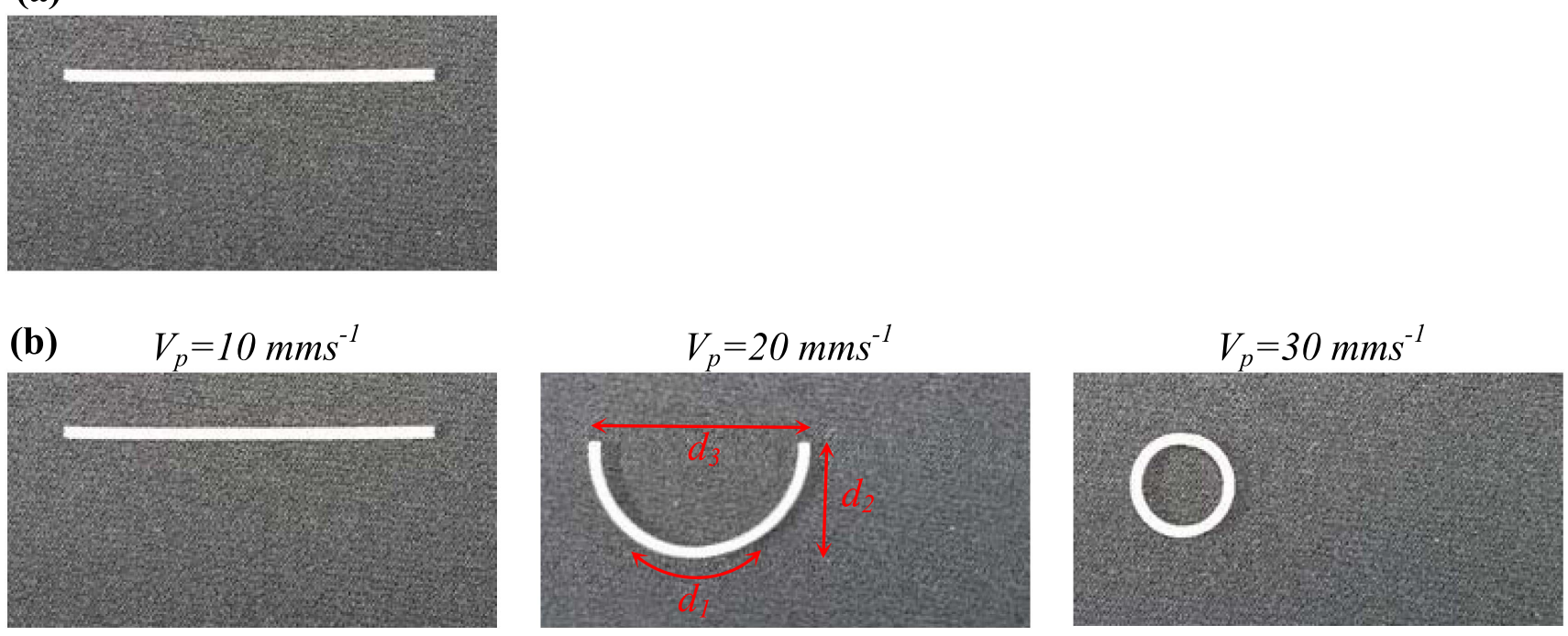

Figure 6. An illustration of dual SMP via hot programming 4D printing with different speeds: configuration of the beams after printing (a) and heating-cooling (b) processes.

straight beams $(34 \times 1.6 \times 1 \mathrm{~mm})$ as key primitive elements of smart/metamaterial structures are fabricated by the $3 \mathrm{D}$ printer introduced in section 2.2. Although, as described in section 2.1, six fabrication factors are expected to influence hot programming process, the effect of the printing speed is put into evidence here by fabricating straight beams with three different speeds of $V_{p}=10,30,40 \mathrm{~mm} \mathrm{~s}^{-1}$.

The configuration of the printed straight beams after FDM printing process is illustrated in figure 6(a). The samples are supposed to be programmed during the printing stage. In order to check this possibility, they are heated by dipping into hot water with a prescribed temperature of $85^{\circ} \mathrm{C}$ that is $25^{\circ} \mathrm{C}$ higher than the transition temperature. They are then removed from the hot water and cooled down to room temperature of $23{ }^{\circ} \mathrm{C}$. The configurations of the printed elements after heating-cooling process are demonstrated in figure 6(b). The geometric parameters of the elements including outer length, depth and opening of the mid-surface curved beams $\left(d_{1} \times d_{2} \times d_{3}\right)$ after heating-cooling are measured and reported in table 1 . The experimental results reveal that the FDM printing process enables the elements to change their shape once activated by heating. In fact, the FDM process shows capability of both fabrication and hot programming for dual SME. The shape adaptive transformation can also be categorized as a self-bending feature as the elements are able to bend themselves into another shape. This selfbending may come from an anisotropic pre-strain regime architected during layer-by-layer fabrication coupled with non-uniform hot programming process. When the printed
Table 1. Geometric parameters of the beams with $34 \mathrm{~mm}$ length $4 \mathrm{D}$ printed by different speeds after heating-cooling process.

\begin{tabular}{lccr}
\hline$V_{p}\left(\mathrm{~mm} \mathrm{~s}^{-1}\right)$ & $d_{1}(\mathrm{~mm})$ & $d_{2}$ & \multicolumn{1}{c}{$d_{3}$} \\
\hline 10 & 34.0 & 0.0 & 34.0 \\
30 & 32.4 & 9.8 & 19.4 \\
40 & 28.1 & 7.9 & 0.0 \\
\hline
\end{tabular}

element is heated, it starts to shrink releasing the pre-stain. However, anisotropic distribution of the pre-strain through the thickness of the beam leads to mismatch in free strain recovery and results in flexural bending and positive curvature. In this regard, self-bending toward the building direction may imply that the top layers have more pre-stain than the bottom layers as expected in section 2.1. Finally, the experimental results show that printing speed can play an important role on hot programming and self-bending feature. It is seen that the printing speed of $10 \mathrm{~mm} \mathrm{~s}^{-1}$ has no capability of inducing pre-strain into the material, while higher speed can provide stretching enough for hot programming and inducing pre-strain. In this respect, as objects are printed faster, selfbending becomes more and curvature gets deeper.

Next, feasibility of triple SMP by 4D printing is examined. To this goal, the beams printed with different speeds of $V_{p}=10,30,40 \mathrm{~mm} \mathrm{~s}^{-1}$ are programmed in a cold manner. The cold programming involves bending the printed beams downward to make a $360^{\circ}$ circle with external diameter of $\approx 12 \mathrm{~mm}$ at a low temperature of $23{ }^{\circ} \mathrm{C}$ and then releasing 


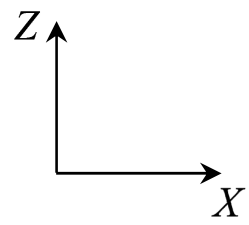

(a)

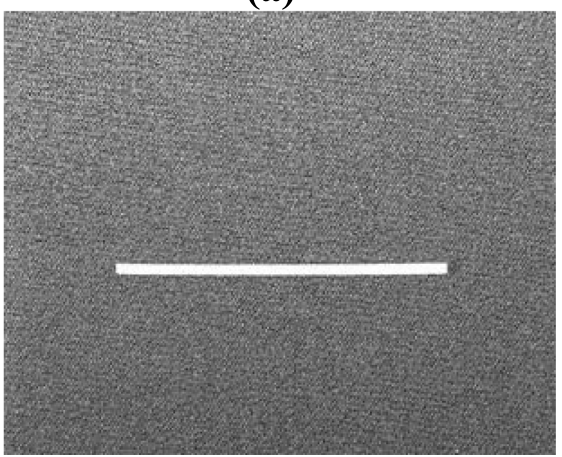

(b)
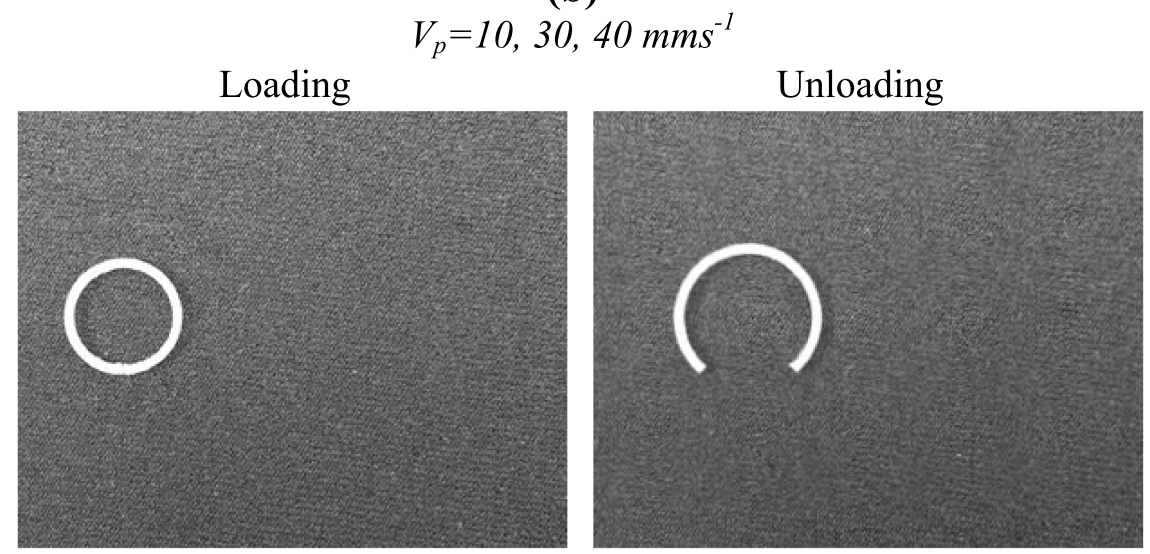

(c)
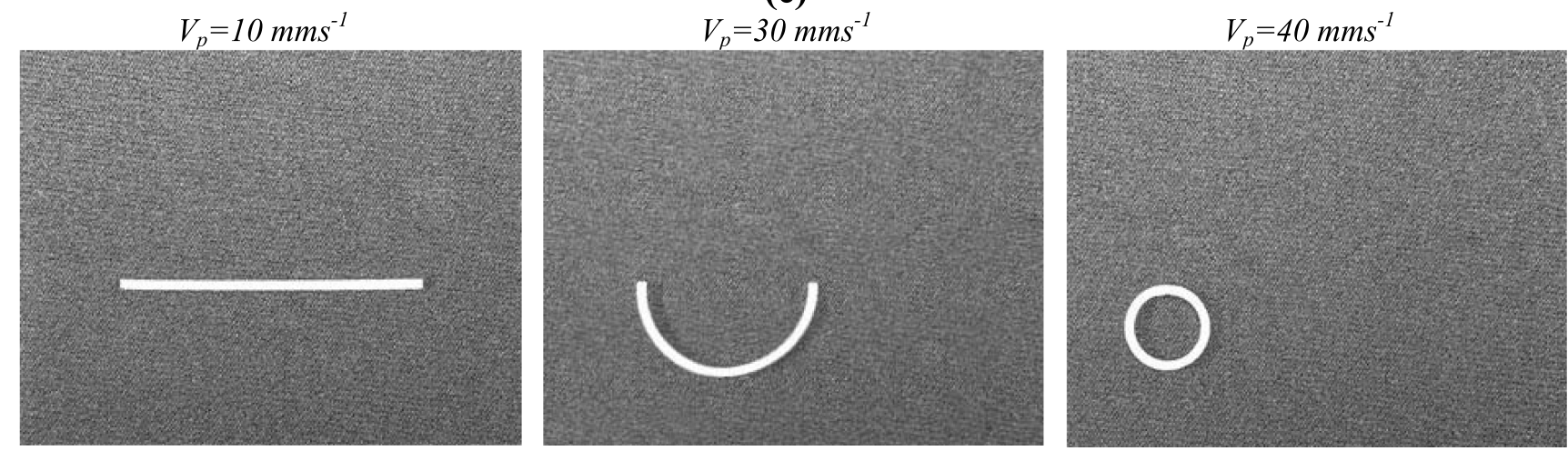

Figure 7. Experimental illustration of triple SMP via hot programming 4D printing with different speeds and cold programming: configuration of the beams after printing (a), cold programming (b) and heating-cooling (c) processes.

them. The configuration of the printed beams after printing and cold programming processes is illustrated in figures 7 (a) and (b). It is worth mentioning that all beams printed with different speeds having different pre-strain regimes become an arch with negative curvature and experience a similar second temporary shape as shown in figure 7(b). It may imply that the pre-strain regime of the material from printing process does not affect cold programming process and consequently the plastic strain regime. The curved beams are then heated by immersing into hot water with a prescribed temperature of $85^{\circ} \mathrm{C}$ and later removed and cooled down to $23{ }^{\circ} \mathrm{C}$. Figure 7(c) shows their configurations after heating-cooling process. Table 2 also includes geometric parameters of the elements after loading, unloading and heating-cooling process. It should be mentioned that numerical results as reported in table 2 would be obtained from the future simulations in section 4. Comparing the results in figures 6(b) and 7(c) reveals that the samples experienced cold programming return toward the original shape. In fact, the printed samples with a negative curvature gradually return back to the first flat 
Table 2. Geometric parameters of the beams with $34 \mathrm{~mm}$ length 4D printed by different speeds after cold programming and heating-cooling processes.

\begin{tabular}{|c|c|c|c|c|c|c|c|c|c|c|}
\hline \multirow[b]{2}{*}{ Method } & \multirow[b]{2}{*}{$V_{p}\left(\mathrm{~mm} \mathrm{~s}^{-1}\right)$} & \multicolumn{3}{|c|}{ Loading } & \multicolumn{3}{|c|}{ Unloading } & \multicolumn{3}{|c|}{ Heating-cooling } \\
\hline & & $d_{1}(\mathrm{~mm})$ & $d_{2}$ & $d_{3}$ & $d_{1}$ & $d_{2}$ & $d_{3}$ & $d_{1}$ & $d_{2}$ & $d_{3}$ \\
\hline \multirow[t]{3}{*}{ Experiment } & 10 & 37.4 & 11.1 & 0.0 & 36.7 & 12.5 & 10.2 & 34.0 & 0.0 & 34.0 \\
\hline & 30 & & & & & & & 32.4 & 9.8 & 19.4 \\
\hline & 40 & & & & & & & 28.1 & 7.9 & 0.0 \\
\hline \multirow[t]{3}{*}{ Simulation } & 10 & 37.10 & 10.97 & 0.00 & 36.40 & 12.39 & 10.04 & 34.00 & 0.00 & 34.00 \\
\hline & 30 & & & & & & & 32.40 & 9.84 & 19.42 \\
\hline & 40 & & & & & & & 28.11 & 7.93 & 0.00 \\
\hline
\end{tabular}

temporary shape and finally recover to the original shape with zero/positive curvatures depending on printing speed. It means all printed samples are able to release all inelastic prestrains and plastic strains induced by hot-cold programming process simply by heating. While the beam printed with low speed shows dual SME by cold programming, the ones printed faster reveal triple plasticity-based SME by 4D printing along with cold programming.

\section{Theoretical model}

The main objective of this section is to establish a quantitative understanding of triple shape memory behaviors of thermoresponsive SMPs fabricated by $4 \mathrm{D}$ printing in the large deformation regime. A 3D phenomenological macroscopic model is derived in section 3.1 to replicate thermo-mechanical features observed experimentally in the 4D printed SMPs. It is developed within the framework of continuum thermodynamics with internal variables. Afterwards, governing equations of equilibrium are established in section 3.2 for the 4D printed SMP structures and then solved by FE method through an elastic-predictor plastic-corrector return map procedure.

\subsection{Material model}

3.1.1. Preliminaries. As revealed by the experiments, 3D printed SMPs may experience large strains. It is assumed that a material point in the reference configuration at point $\boldsymbol{X}$ moves to a spatial point $\boldsymbol{x}$. The displacement, $\boldsymbol{u}$, and deformation gradient, $\boldsymbol{F}$, representing tangent of a general nonlinear mapping, are defined as:

$$
\begin{gathered}
\boldsymbol{u}=\boldsymbol{x}-\boldsymbol{X}, \\
\boldsymbol{F}=\frac{\partial \boldsymbol{x}}{\partial \boldsymbol{X}} .
\end{gathered}
$$

The determinant of $\boldsymbol{F}, J=\operatorname{det}(\boldsymbol{F})$, indicates material volume change during deformation assumed to be nonsingular.
The right Cauchy-Green deformation tensor, $\boldsymbol{C}$, can be defined in terms of $\boldsymbol{F}$ as:

$$
\boldsymbol{C}=\boldsymbol{F}^{T} \boldsymbol{F} .
$$

Consequently, the Green-Lagrange strain tensor, $\boldsymbol{E}$, is expressed as:

$$
\boldsymbol{E}=\frac{1}{2}(\boldsymbol{C}-\boldsymbol{I})
$$

where $\boldsymbol{I}$ indicates the second-order identity tensor.

Finally, relationship between the second Piola-Kirchhoff stress tensor $\boldsymbol{S}$ and the Cauchy stress $\boldsymbol{\sigma}$ can be expressed as:

$$
\boldsymbol{S}=J \boldsymbol{F}^{-1} \boldsymbol{\sigma} \boldsymbol{F}^{-T} .
$$

SMPs show a mixture of glassy and rubbery phases. The volume fractions of the glassy and rubbery phases are characterized by the scalar variables of $\chi_{g}$ and $\chi_{r}$, respectively, and their summation should be unity $\chi_{g}+\chi_{r}=1$. This constraint implies that there is just one independent volume fraction through $\chi_{r}=1-\chi_{g}$. As a generally well-known assumption, $\eta_{g}$ is assumed to be only a function of temperature variable, $T$, i.e., $\chi_{g}=\chi_{g}(T)$. Henceforth, subscripts ' $g$ ' and ' $r$ ' denote glassy and rubbery phases, respectively.

The total deformation gradient $\boldsymbol{F}$ is assumed to be the same for both glassy and rubbery phases defined as:

$$
\boldsymbol{F}=\boldsymbol{F}_{g t}=\boldsymbol{F}_{r t}
$$

It easily implies that

$$
\boldsymbol{C}=\boldsymbol{C}_{g t}=\boldsymbol{C}_{r t}
$$

and means that rubbery to glassy phases in SMPs are linked to each other in a parallel manner. The total deformation gradient of the glassy phase $\boldsymbol{F}_{g t}$ is then considered to be a combination of active glassy phase contribution, $\boldsymbol{F}_{g a}$, and a frozen contribution, $\boldsymbol{F}_{g f}$ that can be separated via a multiplicative decomposition scheme. It is formulated as:

$$
\boldsymbol{F}_{g t}=\boldsymbol{F}_{g a} \boldsymbol{F}_{g f}
$$

The frozen deformation gradient $\boldsymbol{F}_{g f}$ represents deformation induced by hot programming that can be stored temporarily at low temperatures and released upon heating over the transition temperature. The SMP behavior during 
cold programming is considered to be elasto-plastic. Therefore, a multiplicative decomposition of active glassy phase deformation gradient $\boldsymbol{F}_{g a}$ into an elastic part $\boldsymbol{F}_{g e}$, characterized with respect to an middle configuration, and an plastic part $\boldsymbol{F}_{g p}$ related to the reference configuration is adopted as:

$$
\boldsymbol{F}_{g a}=\boldsymbol{F}_{g e} \boldsymbol{F}_{g p} .
$$

Mechanical behavior of the rubbery phase is assumed to be hyper-elastic. Therefore, total deformation gradient of the glassy phase $\boldsymbol{F}_{g t}$ coincides with the hyper-elastic part as:

$$
\boldsymbol{F}_{r t}=\boldsymbol{F}_{r e} .
$$

Considering Green deformation tensor defined in (2) and total deformation gradient of the glassy phase $\boldsymbol{F}_{g t}$ expressed in (5)-(8), one obtains:

$$
\boldsymbol{C}=\boldsymbol{F}_{g f}^{T} \boldsymbol{F}_{g p}^{T} \boldsymbol{F}_{g e}^{T} \boldsymbol{F}_{g e} \boldsymbol{F}_{g p} \boldsymbol{F}_{g f} .
$$

Consequently, elastic Green deformation tensor of the glassy phase can be expressed in terms of Green deformation tensor as:

$$
\boldsymbol{C}_{g e}=\boldsymbol{F}_{g p}^{-T} \boldsymbol{F}_{g f}^{-T} \boldsymbol{C} \boldsymbol{F}_{g f}^{-1} \boldsymbol{F}_{g p}^{-1}
$$

Obviously, elastic Green deformation tensor of the rubbery phase can be written as:

$$
\boldsymbol{C}_{r e}=\boldsymbol{C} \text {. }
$$

3.1.2. Helmholtz free energy function and constitutive relations. The model assumes the Green deformation tensor $\boldsymbol{C}$ and the absolute temperature $T$ as control variables while $\boldsymbol{C}_{g p}\left(=\boldsymbol{F}_{g p}^{T} \boldsymbol{F}_{g p}\right)$ is considered as internal variable. A Helmholtz specific free energy function, $\Psi$, for an SMP material with rubbery and glassy phases is adopted. It can be additively expressed adopting the rule of mixtures as:

$$
\Psi=\chi_{g} \Psi_{g}\left(\boldsymbol{C}_{g e}, \boldsymbol{C}_{g p}, T\right)+\left(1-\chi_{g}\right) \Psi_{r}\left(\boldsymbol{C}_{r e}, T\right) .
$$

Consequently, free energy functions of rubbery and glassy phases can be written as:

$$
\begin{gathered}
\Psi_{g}=\frac{1}{\rho_{0}} \psi_{g e}\left(I_{1 g e}, I_{2 g e}\right)+\frac{1}{\rho_{0}} \psi_{g \mathrm{th}}\left(I_{1 g}, T\right) \\
+\psi_{g p}\left(I_{1 g p}, I_{2 g p}\right)+\psi_{g \mathrm{ch}}(T), \\
\Psi_{r}=\frac{1}{\rho_{0}} \psi_{r e}\left(I_{1 r e}, I_{2 r e}, I_{3 r e}\right)+\frac{1}{\rho_{0}} \psi_{r \mathrm{th}}\left(I_{1 r}, T\right)+\psi_{r \mathrm{ch}}(T),
\end{gathered}
$$

where $\rho_{0}$ is the reference density. $\psi_{i e}(i=g, r)$ indicates elastic strain energy of the glassy and rubbery phases while $\psi_{g p}$ is related to the plastic contribution of the glassy phase. Also, $\psi_{i \text { th }}(i=g, r)$ is the free energy associated to thermal expansion with respect to the a reference temperature, $T_{0}$, while $\psi_{i \text { ch }}(i=g, r)$ is chemical energy. Moreover, $I_{1 i j}, I_{2 i j}$ and $I_{3 i j}(i=g, r ; j=e, p)$ signify the first, second and the third invariant of the Green deformation tensor.

Adopting Saint-Venant-Kirchhoff strain energy function for the glassy phase, $\psi_{g e}$ can be expressed as:

$\psi_{g e}=\left(\frac{\lambda}{8}+\frac{\mu}{4}\right)\left(I_{1 g e}-3\right)^{2}+\frac{\mu}{2}\left(2\left(I_{1 g e}-3\right)-\left(I_{2 g e}-3\right)\right)$,

where $\lambda$ and $\mu$ are Lamè constants.

The Mooney-Rivlin strain energy function is also adopted for the rubbery phase as:

$\psi_{r e}=a_{1}\left(I_{3 r e}^{-1 / 3} I_{1 r e}-3\right)+a_{2}\left(I_{3 r e}^{-2 / 3} I_{2 r e}-3\right)+b_{1}\left(I_{3 r e}^{1 / 2}-1\right)^{2}$

with $a_{1}$ and $a_{2}$ are material constants related to the distortional response while $b_{1}$ is a material constant associated to the volumetric response.

The configurational energy due to the plastic contribution of the glassy phase can be written as:

$$
\psi_{g p}=\frac{1}{8} h\left(\left(I_{1 g p}-3\right)^{2}+4\left(I_{1 g p}-3\right)-2\left(I_{2 g p}-3\right)\right),
$$

where $h$ is a material parameter that governs initial hardening during plastic deformation in the glassy phase. Free energy contributions of $\psi_{i \text { th }}$ and $\psi_{i \text { ch }}(i=g, r)$ can also be expressed as:

$$
\begin{gathered}
\psi_{i \mathrm{th}}=-\frac{3}{2} \alpha_{i} \kappa_{i}\left(I_{1 i}-3\right)\left(T-T_{0}\right), \\
\psi_{i \mathrm{ch}}=u_{0 i}-T s_{0 i}+\mathrm{c}_{i}\left[\left(T-T_{0}\right)-T \ln \left(T / T_{0}\right)\right],
\end{gathered}
$$

where $\alpha_{i}(i=g, r)$ denotes the thermal expansion coefficient; $\kappa_{i}(i=g, r)$ is the bulk modulus; $u_{0 i}$ and $s_{0 i}(i=g, r)$ mean the specific internal energy and entropy at the equilibrium temperature $T_{0}$; while $c_{i}(i=g, r)$ is the specific heat at constant volume.

In order to establish constitutive equations, the second law of thermodynamics in the sense of Clausius-Duhem inequality should be fulfilled. Assuming the positiveness of the thermal dissipation [22], mechanical dissipation inequality can be written as:

$$
D_{m}=\frac{1}{2 \rho_{0}} \boldsymbol{S}: \dot{\boldsymbol{C}}-(\dot{\Psi}+s \dot{T}) \geqslant 0,
$$

where $s$ is the specific entropy.

Taking the time derivative of the Helmholtz free energy (13), the mechanical dissipation inequality (19) can be 
rewritten as:

$$
\begin{aligned}
\rho_{0} D_{m}= & \frac{1}{2}\left(\chi_{g}\left(\boldsymbol{S}_{g}-2\left(\frac{\partial \psi_{g e}}{\partial I_{1 g e}} \frac{\partial I_{1 g e}}{\partial \boldsymbol{C}}+\frac{\partial \psi_{g e}}{\partial I_{2 g e}} \frac{\partial \boldsymbol{I}_{2 g e}}{\partial \boldsymbol{C}}+\frac{\partial \psi_{g \mathrm{th}}}{\partial \boldsymbol{I}_{1 g}} \frac{\partial I_{1 g}}{\partial \boldsymbol{C}}\right)\right)\right. \\
& +\left(1-\chi_{g}\right)\left(\boldsymbol{S}_{r}-2\left(\frac{\partial \psi_{r e}}{\partial I_{1 r e}} \frac{\partial I_{1 r e}}{\partial \boldsymbol{C}}+\frac{\partial \psi_{r e}}{\partial I_{2 r e}} \frac{\partial \boldsymbol{I}_{2 r e}}{\partial \boldsymbol{C}}\right.\right. \\
& \left.\left.\left.+\frac{\partial \psi_{r e}}{\partial I_{3 r e}} \frac{\partial I_{3 r e}}{\partial \boldsymbol{C}}+\frac{\partial \psi_{r \mathrm{th}}}{\partial \boldsymbol{I}_{1 r}} \frac{\partial \boldsymbol{I}_{1 r}}{\partial \boldsymbol{C}}\right)\right)\right): \dot{\boldsymbol{C}} \\
& -\chi_{g}\left(\left(\frac{\partial \psi_{g e}}{\partial I_{1 g e}} \frac{\partial I_{1 g e}}{\partial \boldsymbol{C}_{g p}}+\frac{\partial \psi_{g e}}{\partial I_{2 g e}} \frac{\partial I_{2 g e}}{\partial \boldsymbol{C}_{g p}}\right.\right. \\
& \left.+\rho_{0} \frac{\partial \psi_{g p}}{\partial \boldsymbol{I}_{g p}} \frac{\partial \boldsymbol{I}_{1 g p}}{\partial \boldsymbol{C}_{g p}}+\rho_{0} \frac{\partial \psi_{g p}}{\partial I_{2 g p}} \frac{\partial \boldsymbol{I}_{2 g p}}{\partial \boldsymbol{C}_{g p}}\right): \dot{\boldsymbol{C}}_{g p} \\
& -\left(\chi_{g}\left(\rho_{0} s_{g}+\frac{\partial \psi_{g \mathrm{th}}}{\partial T}+\rho_{0} \frac{\partial \psi_{g \mathrm{ch}}}{\partial T}\right)\right. \\
& \left.+\left(1-\chi_{g}\right)\left(\rho_{0} \boldsymbol{s}_{r}+\frac{\partial \psi_{r \mathrm{~h}}}{\partial T}+\rho_{0} \frac{\partial \psi_{r \mathrm{ch}}}{\partial T}\right)\right) \dot{T} \geqslant 0,
\end{aligned}
$$

where $\boldsymbol{S}_{g}, \boldsymbol{S}_{r}$ and $s_{g}, s_{r}$ are glassy and rubbery components of the second Piola-Kirchhoff stress tensor and the entropy defined through a rule of mixtures as:

$$
\begin{gathered}
\boldsymbol{S}=\chi_{g} \boldsymbol{S}_{g}+\left(1-\chi_{g}\right) \boldsymbol{S}_{r}, \\
s=\chi_{g} \boldsymbol{s}_{g}+\left(1-\chi_{g}\right) s_{r} .
\end{gathered}
$$

Following a standard argument [22], and implying sufficient conditions for the second Piola-Kirchhoff stress tensor and the entropy, the state equations are derived as:

$$
\begin{aligned}
\boldsymbol{S}_{g} & =2\left(\frac{\partial \psi_{g e}}{\partial \boldsymbol{I}_{g e}} \frac{\partial \boldsymbol{I}_{1 g e}}{\partial \boldsymbol{C}}+\frac{\partial \psi_{g e}}{\partial \boldsymbol{I}_{2 g e}} \frac{\partial \boldsymbol{I}_{2 g e}}{\partial \boldsymbol{C}}+\frac{\partial \psi_{g \mathrm{th}}}{\partial \boldsymbol{I}_{1 g}} \frac{\partial I_{1 g}}{\partial \boldsymbol{C}}\right), \\
\boldsymbol{S}_{r} & =2\left(\frac{\partial \psi_{r e}}{\partial \boldsymbol{I}_{1 r e}} \frac{\partial \boldsymbol{I}_{1 r e}}{\partial \boldsymbol{C}}+\frac{\partial \psi_{r e}}{\partial \boldsymbol{I}_{2 r}} \frac{\partial \boldsymbol{I}_{2 r e}}{\partial \boldsymbol{C}}+\frac{\partial \psi_{r e}}{\partial \boldsymbol{I}_{3 r e}} \frac{\partial I_{3 r e}}{\partial \boldsymbol{C}}+\frac{\partial \psi_{r \mathrm{th}}}{\partial \boldsymbol{I}_{1 r}} \frac{\partial \boldsymbol{I}_{1 r}}{\partial \boldsymbol{C}}\right), \\
\boldsymbol{S}_{g} & =-\frac{1}{\rho_{0}} \frac{\partial \psi_{g \mathrm{th}}}{\partial T}-\frac{\partial \psi_{g \mathrm{ch}}}{\partial T}, \\
\boldsymbol{S}_{r} & =-\frac{1}{\rho_{0}} \frac{\partial \psi_{r \mathrm{th}}}{\partial T}-\frac{\partial \psi_{r \mathrm{ch}}}{\partial T}
\end{aligned}
$$

and the mechanical dissipation inequality (20) reads

$$
\boldsymbol{X}_{g p}: \dot{\boldsymbol{C}}_{g p} \geqslant 0
$$

where $\boldsymbol{X}_{g p}$ represents the thermo-dynamical dissipative force associated to the plastic velocity gradient and defined as:

$$
\begin{aligned}
\boldsymbol{X}_{g p}= & -\left(\frac{\partial \psi_{g e}}{\partial I_{1 g e}} \frac{\partial I_{1 g e}}{\partial \boldsymbol{C}_{g p}}+\frac{\partial \psi_{g e}}{\partial I_{2 g e}} \frac{\partial I_{2 g e}}{\partial \boldsymbol{C}_{g p}}+\rho_{0} \frac{\partial \psi_{g p}}{\partial I_{1 g p}} \frac{\partial I_{1 g p}}{\partial \boldsymbol{C}_{g p}}\right. \\
& \left.+\rho_{0} \frac{\partial \psi_{g p}}{\partial I_{2 g p}} \frac{\partial I_{2 g p}}{\partial \boldsymbol{C}_{g p}}\right) .
\end{aligned}
$$

Since plastic deformation in glassy phase is isotropic, deviatoric component of $\boldsymbol{X}_{g p}$ signified as $\boldsymbol{X}_{g p}^{D}$ is assumed to contribute in plastic evolution.

Taking derivative of $I_{1 i j}, I_{2 i j}, I_{3 i j}(i=g, r ; j=e, p)$ with respect to $\boldsymbol{C}, \boldsymbol{C}_{g p}$, and $\psi_{i j}, \psi_{i \text { th }}(i=g, r ; j=e, p)$ with respect to $I_{1 i j}, I_{2 i j}, I_{3 i j}, T$, and considering incompressibility condition for the rubbery phases, $J_{r e}=1, S, s$ and
$\boldsymbol{X}_{g p}$ can be derived as:

$$
\begin{aligned}
& \boldsymbol{S}_{g}=\left(\left(\frac{\lambda}{2}+\mu\right)\left(I_{1 g e}-3\right)+2 \mu\right) \boldsymbol{C}_{g p f}^{-1}-\mu\left(I_{1 g e} \boldsymbol{I}\right. \\
&\left.-\boldsymbol{C}_{g p f}^{-1} \boldsymbol{C}\right) \boldsymbol{C}_{g p f}^{-1}-3 \alpha_{g} \kappa_{g}\left(T-T_{0}\right) \boldsymbol{I}, \\
& \boldsymbol{S}_{r}= 2\left(a_{1}+a_{2} I_{1 r}\right) \boldsymbol{I}-2 a_{2} \boldsymbol{C}-\frac{2}{3}\left(a_{1} I_{1 r}+2 a_{2} I_{2 r}\right) \boldsymbol{C}^{-1}, \\
& s_{g}=\frac{3}{2 \rho_{0}} \alpha_{g} \kappa_{g}\left(I_{1 g e}-3\right)+s_{0 g}+c_{g} \ln \left(T / T_{0}\right), \\
& s_{r}=\frac{3}{2 \rho_{0}} \alpha_{r} \kappa_{r}\left(I_{1 r e}-3\right)+s_{0 r}+c_{r} \ln \left(T / T_{0}\right), \\
& \boldsymbol{X}_{g p}=\left(\left(\frac{\lambda}{4}+\frac{\mu}{2}\right)\left(I_{1 g e}-3\right)+\mu\right) \boldsymbol{C}_{g p}^{-1} \boldsymbol{C}_{t f} \boldsymbol{C}_{g p}^{-1}-\frac{\mu}{2}\left(I_{1 g e}\right. \\
&\left.\quad-\boldsymbol{C}_{g p}^{-1} \boldsymbol{C}_{t f}\right) \boldsymbol{C}_{g p}^{-1} \boldsymbol{C}_{t f} \boldsymbol{C}_{g p}^{-1}-\frac{1}{4} \varpi\left(\boldsymbol{C}_{g p}-\boldsymbol{I}\right),
\end{aligned}
$$

where

$$
\begin{aligned}
& \boldsymbol{C}_{g p f}=\boldsymbol{F}_{g f} \boldsymbol{C}_{g p} \boldsymbol{F}_{g f}^{T}, \\
& \boldsymbol{C}_{t f}=\boldsymbol{F}_{g f}^{-T} \boldsymbol{C F}_{g f}^{-1}
\end{aligned}
$$

and $\varpi=\rho_{0} h$ is considered as a relative stress.

3.1.3. Evolution laws and limit functions. In this section, evolution equations for $\boldsymbol{C}_{g p}$ as well as $\chi_{g}, \boldsymbol{F}_{g f}$ are determined. In order to satisfy the second law of thermodynamics during plastic deformation of the glassy phase, the evolution equation for $\boldsymbol{C}_{g p}$ is given as:

$$
\dot{\boldsymbol{C}}_{g p}=\dot{\beta} \boldsymbol{X}_{g p}^{D} /\left\|\boldsymbol{X}_{g p}^{D}\right\|,
$$

where $\beta$ is non-negative consistency parameter. Using equation (27), the mechanical dissipation inequality (23) can be rewritten as:

$$
\dot{\beta}\left\|\boldsymbol{X}_{g p}^{D}\right\| \geqslant 0
$$

In order to govern plastic evolution in glassy phase, the following limit function is introduced:

$$
L_{g p}=\left\|\boldsymbol{X}_{g p}^{D}\right\|-Y_{g p}
$$

where $Y_{g p}$ represents a function that directs the kinetics of mechanically induced plastic deformation. The evolution of $\boldsymbol{C}_{g p}$ is constrained by the classical Kuhn-Tucker conditions as:

$$
L_{g p} \leqslant 0, \quad \dot{\beta} \geqslant 0, \quad L_{g p} \dot{\beta}=0 .
$$

$\boldsymbol{C}_{g p}$ can evolve under mechanical loading at low temperatures $\left(T \leqslant T_{l}\right)$ or during cooling in the transition regime $\left(T_{l}<T<T_{h}\right)$. It is also recovered by heating within transition range, while it is not present beyond $T_{h}$. Therefore, from a mathematical point of view, plastic deformation evolution can 
be described in various temperature domains as:

$\left\{\begin{array}{l}\text { if }\left(T \leqslant T_{l}\right) \text { or }\left(T_{l}<T<T_{h}, \dot{T} \leqslant 0\right) \text { then } C_{g p} \text { evolves via equation (27) } \\ \text { if }\left(T_{l}<T<T_{h}, \dot{T} \geqslant 0\right) \text { then } C_{g p} \text { remains constant } \\ \text { if }\left(T \geqslant T_{h}\right) C_{g p} \text { is set } I .\end{array}\right.$

Regarding its solution, the evolutive constitutive model can be solved by implementing an incremental-iterative procedure on the basis of the elastic-predictor plasticcorrector return mapping scheme details of which can be found in [23]. An elastic trial state is evaluated for $\boldsymbol{C}_{g p}$ and a trial value of $L_{g p}$ is calculated to investigate the admissibility of the trial state. If $L_{g p}$ becomes negative then the SMP behavior is elastic and the trial state is admissible. Otherwise, the step is plastic and $\boldsymbol{C}_{g p}$ needs to be updated by discretizing equation (27) via the explicit forward-Euler integration rule and solving nonlinear system of algebraic equations. This can be done by implementing an iterative method such as Newton-Raphson method [23, 24].

The evolution relation for the frozen deformation gradient in the glassy phase is assumed as:

$$
\left\{\begin{array}{l}
\text { if }\left(T \geqslant T_{h}\right) \text { then } \boldsymbol{F}_{g f}=c_{g f}(\boldsymbol{F}-\boldsymbol{I})+\boldsymbol{I} \\
\text { otherwise } \boldsymbol{F}_{g f} \text { remains constant, }
\end{array}\right.
$$

where $c_{g f}$ is a material parameter that control the shape-fixing during hot programming process. It ranges in [0 1]. All the deformation is frozen when $c_{g f}=1$ through $\boldsymbol{F}_{g f}=\boldsymbol{F}$ while $0 \leqslant c_{g f}<1$ leads an imperfect/null shape-fixing.

In conjunction with the evolution of $\chi_{g}$, it is directly related to the SMP phase transformation and can be obtained by matching experimental DMA results. An explicit interpolation formula is introduced in terms of trigonometric functions to fit storage modulus extracted from DMA data. It is formulated as:

$$
\chi_{g}=-\frac{\tanh \left(\gamma_{1} T_{g}-\gamma_{2} T\right)-\tanh \left(\gamma_{1} T_{g}-\gamma_{2} T_{h}\right)}{\tanh \left(\gamma_{1} T_{g}-\gamma_{2} T_{h}\right)-\tanh \left(\gamma_{1} T_{g}-\gamma_{2} T_{l}\right)},
$$

where parameters $\gamma_{1}$ and $\gamma_{2}$ are chosen to fit DMA curve.

\subsection{Structural model}

In this section, governing equations of equilibrium of $4 \mathrm{D}$ printed triple SME structures under thermo-mechanical loadings are extracted by means of the principle of minimum total potential energy and then solved by an in-house FE approach. In this respect, the total Lagrangian description is adopted to determine deformation and stress states in the current problem. In this formulation, all quantities are measured with respect to the original undeformed configuration.

While SMP constitutive equations have been introduced in a tensorial form, the FE formulation is established here in a vectorial framework that is convenient for computational programming. To this end, by using some algebraic manipulations, stress-strain relationship, equations (21a) and (25a), can be expressed in a matrix-vector format as:

$$
\boldsymbol{s}=\boldsymbol{Q}\left(\boldsymbol{F}_{g f}, \boldsymbol{C}_{g p}, T\right)\left(\boldsymbol{e}-\boldsymbol{p}\left(\boldsymbol{F}_{g f}, \boldsymbol{C}_{g p}, T\right)\right),
$$

where $\boldsymbol{s}$ and $\boldsymbol{e}$ are vectors of the second Piola-Kirchhoff stress and Green-Lagrange strain while the matrix $\boldsymbol{Q}$ and the vector $\boldsymbol{p}$ are functions of temperature and components of $\boldsymbol{F}_{g f}$ and $\boldsymbol{C}_{g p}$.

The nonlinear strain-displacement kinematic relation can be written as:

$$
e=\left(L+\frac{1}{2} N\right) v,
$$

where $v$ signifies the gradient displacement defined as:

$$
\boldsymbol{v}=\left\{\begin{array}{l}
\boldsymbol{u}_{, X} \\
\boldsymbol{u}_{, Y} \\
\boldsymbol{u}_{, Z}
\end{array}\right\}
$$

in terms of displacement field vector of

$$
\boldsymbol{u}=\left\{\begin{array}{c}
u \\
v \\
w
\end{array}\right\}
$$

in which $u, v$ and $w$ indicate displacement components of a typical point within spatial printed structure along $X, Y, Z$ axes, respectively. Linear and nonlinear operator matrices of $\boldsymbol{L}$ and $\boldsymbol{N}$ are also defined by:

$$
\boldsymbol{L}=\left[\begin{array}{ccc}
\boldsymbol{i}_{1}^{T} & \mathbf{0} & \mathbf{0} \\
\mathbf{0} & \boldsymbol{i}_{2}^{T} & \mathbf{0} \\
\mathbf{0} & \mathbf{0} & \boldsymbol{i}_{3}^{T} \\
\boldsymbol{i}_{2}^{T} & \boldsymbol{i}_{1}^{T} & \mathbf{0} \\
\boldsymbol{i}_{3}^{T} & \mathbf{0} & \boldsymbol{i}_{1}^{T} \\
\mathbf{0} & \boldsymbol{i}_{3}^{T} & \boldsymbol{i}_{2}^{T}
\end{array}\right] \quad \boldsymbol{N}=\left[\begin{array}{ccc}
\boldsymbol{u}_{, X}^{T} & \mathbf{0} & \mathbf{0} \\
\mathbf{0} & \boldsymbol{u}_{, Y}^{T} & \mathbf{0} \\
\mathbf{0} & \mathbf{0} & \boldsymbol{u}_{, Z}^{T} \\
\boldsymbol{u}_{, Y}^{T} & \boldsymbol{u}_{, X}^{T} & \mathbf{0} \\
\boldsymbol{u}_{, Z}^{T} & \mathbf{0} & \boldsymbol{u}_{, X}^{T} \\
\mathbf{0} & \boldsymbol{u}_{, Z}^{T} & \boldsymbol{u}_{, Y}^{T}
\end{array}\right]
$$

where

$$
i_{1}=\left\{\begin{array}{l}
1 \\
0 \\
0
\end{array}\right\}, i_{2}=\left\{\begin{array}{l}
0 \\
1 \\
0
\end{array}\right\}, i_{3}=\left\{\begin{array}{l}
0 \\
0 \\
1
\end{array}\right\} .
$$

Equilibrium equations are derived from the static version of the principle of virtual work known as the principle of the minimum total potential energy. It is formulated as:

$$
\delta \Pi=\delta U-\delta W=0,
$$

where $\delta$ signifies the variational operator. Also $\delta \Pi$ denotes variation of the total potential energy of the printed object while $\delta U$ and $\delta W$ are internal virtual work stored in the structure due to the actual stress $s$ in moving through the virtual strain $\delta \boldsymbol{e}$, and external virtual work done by applied loads $\boldsymbol{t}$ in moving through the virtual displacement $\delta \boldsymbol{u}$, respectively. These virtual energies in the absence of body forces can be defined as:

$$
\begin{aligned}
& \delta U=\int_{V} \delta \boldsymbol{e}^{T} \boldsymbol{s} \mathrm{d} V, \\
& \delta W=\int_{A} \delta \boldsymbol{u}^{T} \boldsymbol{t} \mathrm{d} A,
\end{aligned}
$$

where $V$ and $A$ indicates volume and surface of the body.

In order to solve the problem, an FE solution is developed using 20-noded serendipity hexahedron element. The 
element has three degrees of freedom per node. Consequently, the displacement field can be approximated in terms of nodal displacements through interpolation functions as:

$$
\boldsymbol{u}=\boldsymbol{M w},
$$

where

$$
\begin{aligned}
& \boldsymbol{M}=\left[\begin{array}{lll}
\boldsymbol{m}_{1} & \cdots & \boldsymbol{m}_{20}
\end{array}\right], \boldsymbol{m}_{i}=\left[\begin{array}{ccc}
m_{i} & 0 & 0 \\
0 & m_{i} & 0 \\
0 & 0 & m_{i}
\end{array}\right], \\
& \boldsymbol{w}=\left\{\begin{array}{c}
\boldsymbol{u}_{1} \\
\vdots \\
\boldsymbol{u}_{20}
\end{array}\right\}, \boldsymbol{u}_{i}=\left\{\begin{array}{c}
u_{i} \\
v_{i} \\
w_{i}
\end{array}\right\}
\end{aligned}
$$

in which $m_{i}(i=1 \ldots 20)$ are quadrilateral shape functions [24] and $\boldsymbol{u}_{i}$ is mechanical displacement field of $i$ th node.

Employing equation (42), the gradient displacement vector $\boldsymbol{v}$ can also be written in terms of $\boldsymbol{w}$ as:

$$
\boldsymbol{v}=\boldsymbol{R} \boldsymbol{w},
$$

where

$$
\boldsymbol{R}=\left[\begin{array}{lll}
\boldsymbol{m}_{1, X} & \cdots & \boldsymbol{m}_{20, X} \\
\boldsymbol{m}_{1, Y} & \cdots & \boldsymbol{m}_{20, Y} \\
\boldsymbol{m}_{1, Z} & \cdots & \boldsymbol{m}_{20, Z}
\end{array}\right] .
$$

Using equation (44), the strain field (35) can be discretized as:

$$
e=\left(L+\frac{1}{2} \bar{N}\right) R w
$$

in which $\bar{N}$ is an explicit function of $\boldsymbol{w}$.

It can be readily shown that virtual strain field becomes:

$$
\delta \boldsymbol{e}=(\boldsymbol{L}+\overline{\boldsymbol{N}}) \boldsymbol{R} \delta \boldsymbol{w} .
$$

Substituting equations (42), (46) and (47) into the principle of minimum total potential energy (40), FE governing equations can be derived as:

$$
\left(\boldsymbol{K}_{0}+\boldsymbol{K}_{l}+\boldsymbol{K}_{n}\right) \boldsymbol{w}=\boldsymbol{f}_{t}+\boldsymbol{f}_{m},
$$

where

$$
\begin{aligned}
& \boldsymbol{K}_{0}=\int_{V}(\boldsymbol{L R})^{T} \boldsymbol{Q}(\boldsymbol{L R}) \mathrm{d} V, \\
& \boldsymbol{K}_{l}=\int_{V}\left(\frac{1}{2}(\boldsymbol{L R})^{T} \boldsymbol{Q}(\overline{\boldsymbol{N}} \boldsymbol{R})+(\overline{\boldsymbol{N}} R)^{T} \boldsymbol{Q}(\boldsymbol{L} \boldsymbol{R})\right) \mathrm{d} V, \\
& \boldsymbol{K}_{n}= \frac{1}{2} \int_{V}(\overline{\boldsymbol{N}} R)^{T} \boldsymbol{Q}(\overline{\boldsymbol{N}} R) \mathrm{d} V, \\
& \boldsymbol{f}_{t}= \int_{V}\left((\boldsymbol{L R})^{T}+(\overline{\boldsymbol{N}} R)^{T}\right) \boldsymbol{Q p} \mathrm{d} V, \\
& \boldsymbol{f}_{m}=\left\{\begin{array}{c}
\boldsymbol{f}_{1} \\
\vdots \\
\boldsymbol{f}_{20}
\end{array}\right\}, \boldsymbol{f}_{i}=\left\{\begin{array}{l}
f_{X-i} \\
f_{Y-i} \\
f_{Z-i}
\end{array}\right\}, i=1 \ldots 20
\end{aligned}
$$

in which $\boldsymbol{K}_{0}$ indicates the constant stiffness matrix while $\boldsymbol{K}_{l}$ and $\boldsymbol{K}_{n}$ signify stiffness matrices that are linearly and quadratically dependent on the nodal displacement vector, respectively. $f_{X-i}, f_{Y-i}$ and $f_{Z-i}$ indicate point forces applied on the $i$ th node along $X, Y$ and $Z$ directions, respectively. $f_{t}$ also denotes the load vector induced by thermal loading and/ or SMP phase transformation. It should be mentioned that, beyond geometrical nonlinearity, the stiffness matrices and thermo-inelastic loading vector are functions of unknown
Table 3. Material parameters from experiments.

\begin{tabular}{lllll}
\hline$\lambda(\mathrm{GPa})$ & $\mu(\mathrm{GPa})$ & $\alpha_{g}\left(10^{-4} \mathrm{~K}^{-1}\right)$ & $\kappa_{g}(\mathrm{MPa})$ & $c_{g f}$ \\
\hline 1.43 & 0.615 & 1 & 1844 & 1 \\
$\varpi(\mathrm{MPa})$ & $Y_{g p}(\mathrm{MPa})$ & $a_{1}, a_{2}(\mathrm{MPa})$ & $\gamma_{1}, \gamma_{2}$ & $T_{l}, T_{h}\left({ }^{\circ} \mathrm{C}\right)$ \\
1 & 24 & $0.55,-0.033$ & $0.15,0.145$ & 23,85 \\
\hline
\end{tabular}

variables $\boldsymbol{F}_{g f}$ and $\boldsymbol{C}_{g p}$. It couples the virtual strain energy to SMP constitutive relations formerly described in section 3.1. In this work, unknown variables $\boldsymbol{F}_{g f}$ and $\boldsymbol{C}_{g p}$ are discretized inside the SMP element by 3D Gauss-Legendre quadrature integration method. Volumetric integrals in equation (49) are numerically integrated by means of $l_{X} \times l_{Y} \times l_{Z}$ discrete grid points along $X, Y, Z$ axes. In conjugation with temperature distribution in the SMP element and evaluation of the volumetric integral including $T$, it is assumed to be raised uniformly through the SMP element volume as a generally well-known assumption.

Finally, equation (48) is used to construct global FE governing equations of equilibrium for a 4D printed structure by assembling technique and applying boundary conditions that results in:

$$
\left(\hat{\boldsymbol{K}}_{0}+\hat{\boldsymbol{K}}_{l}+\hat{\boldsymbol{K}}_{n}\right) \hat{\boldsymbol{w}}=\hat{\boldsymbol{f}}_{t}+\hat{\boldsymbol{f}}_{m} .
$$

This is a nonlinear system of equations in terms of unknown nodal mechanical variables, $\hat{\boldsymbol{w}}$, and unknown internal variables of $\boldsymbol{F}_{g f}$ and $\boldsymbol{C}_{g p}$ distributed inside each SMP element. $\boldsymbol{F}_{g f}$ and $\boldsymbol{C}_{g p}$ can be updated by SMP constitutive equations while they depend on the unknown strain and known temperature fields. This coupling relates structural governing equations to the SMP model making a complicated problem. In order to find a solution for the present problem involving both material and geometrical nonlinearities, an incremental-iterative procedure on the basis of the elasticpredictor plastic-corrector return mapping scheme [23] is implemented. This is also accomplished by using the Newton-Raphson method to trace nonlinear equilibrium path in the finite strain regime details of which can be found elsewhere [24].

\section{Simulations and applications}

The computational tool developed in the previous section is conducted here to simulate triple SMP structures enabled by 4D printing and explore hot-cold programming mechanisms behind this type of material tailoring. Furthermore, it is shown that the 4D printed triple SMPs have great potential in mechanical/bio-medical applications like self-bending gripers/stents and self-shrinking/tightening staples.

First, material properties introduced in the constitutive model need to be determined. The parameters related to the SMP phase transformation including $\gamma_{1}, \gamma_{2}$ and $T_{l}, T_{h}$ in equation (33) are calibrated using experimental DMA data displayed in figure 4 . They are respectively chosen as listed in table 3 to fit DMA curve for Young's modulus as demonstrated in figure 8. It is seen that the trigonometric function 


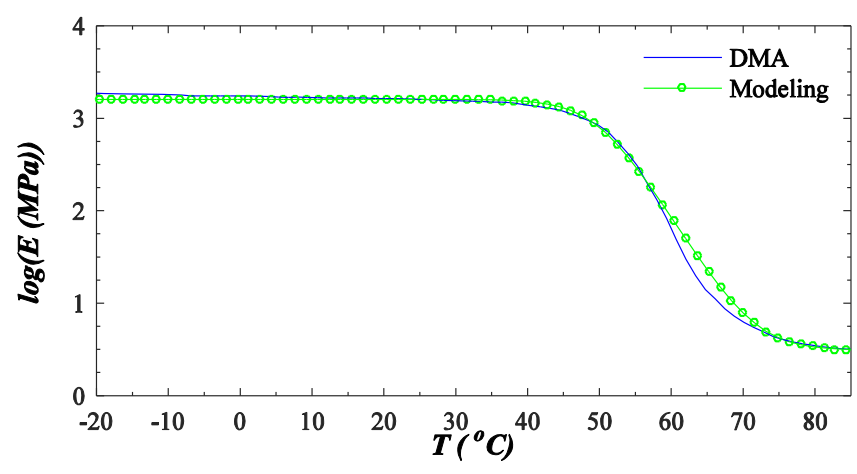

Figure 8. Comparison between model predictions and experimental DMA results for Young's modulus.
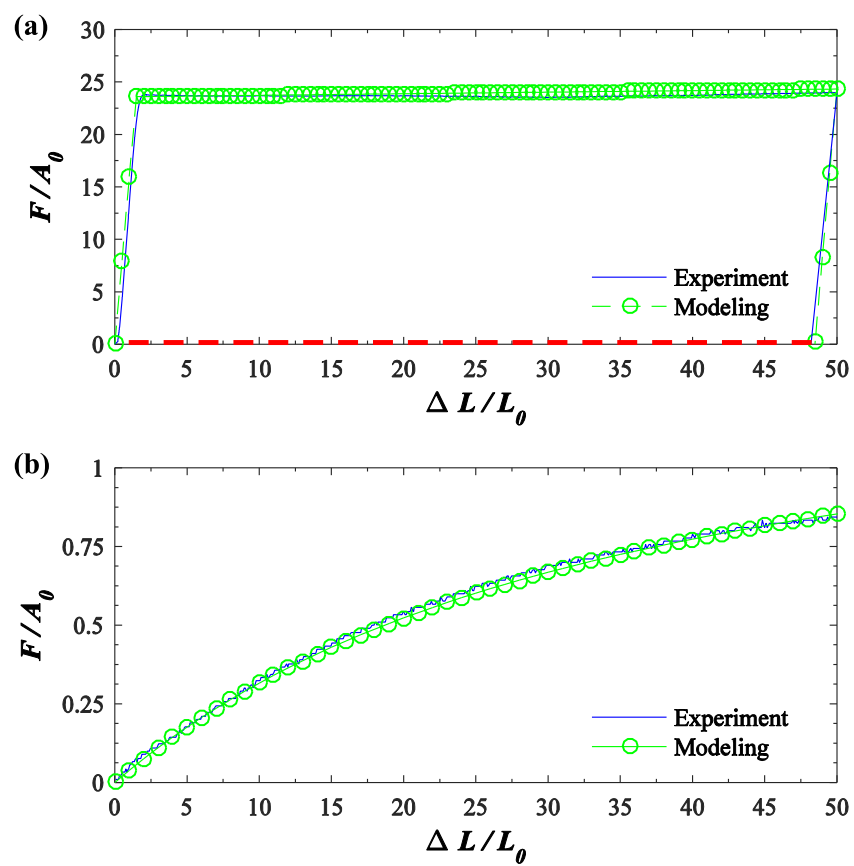

Figure 9. Model predictions for uniaxial tests at temperatures of (a) $23{ }^{\circ} \mathrm{C}$, (b) $85^{\circ} \mathrm{C}$ and comparison with experiments. (for $T=23^{\circ} \mathrm{C}$, experimental and numerical strain recovery induced by the temperature rise is indicated with a red dashed line)

can capture well smooth phase transformation in the printed SMP material. Elastic material properties such as $\lambda, \mu, \alpha_{g}, \kappa_{g}, c_{g f}$ are calibrated by means of small/largestrain uniaxial and thermal tests described in section 2.2 as presented in table 3 . Finally, using uniaxial large-strain experimental tensile tests performed at $23{ }^{\circ} \mathrm{C}$ and $85{ }^{\circ} \mathrm{C}$ as illustrated in figure 5, material parameters of $\varpi, a_{1}, a_{2}, Y_{g p}$ are set as listed in table 3. Model prediction for the tension tests at $23{ }^{\circ} \mathrm{C}$ and $85{ }^{\circ} \mathrm{C}$ are presented figure 9 and compared with experiments. Moreover, the shape recovery under temperature control is examined in figure 9 distinguished by a dashed line. To this end, after loading-unloading at $23{ }^{\circ} \mathrm{C}$, the sample is heated up to $85^{\circ} \mathrm{C}$. Figure 9 shows that the SMP has a temperature-dependent transient behavior from elastoplastic at low temperatures to hyper-elastic at high temperatures. This comparison study reveals that the SMP constitutive model well predicts experimental features such as
Table 4. Anisotropic pre-strain regime (\%) predicted for the SMP elements printed with different speeds.

\begin{tabular}{lccc}
\hline \multirow{2}{*}{ Location } & \multicolumn{3}{c}{$V_{p}\left(\mathrm{~mm} \mathrm{~s}^{-1}\right)$} \\
\cline { 2 - 4 } & 10 & 30 & 40 \\
\hline Upper layer & 0 & 14.2 & 30.75 \\
Middle layers & 0 & 10 & 24 \\
Lower layer & 0 & 5.8 & 17.25 \\
\hline
\end{tabular}

loading/unloading path, nearly flat plastic deformation, plastic shape recovery and nonlinear hyper-elasticity.

The nonlinear structural-material FE solution is now implemented to replicate $4 \mathrm{D}$ printed beams with dual SME and self-folding feature presented in figure 6. The temporary and original shapes of the printed beams characterized in figure 6 and table 1 are considered as inputs and FE analysis is performed to predict the pre-strain range. The pre-strain regime is calculated to be uniform along the printing direction while it has different values at first, middle and end printing layers. The computed values of the pre-strain at these three printing layers are reported in table 4 for different printing speeds. It is seen that, while $3 \mathrm{D}$ printing with a low speed of $10 \mathrm{~mm} \mathrm{~s}^{-1}$ cannot induce any pre-strain, faster printing is able to anisotropically program the material through the-thickness direction. In this respect, the pre-strain value increases from the bottom layer towards the top layer in an almost linear manner, as expected. It can also be found that, the increase in printing speed, $30 \rightarrow 40 \mathrm{~mm} \mathrm{~s}^{-1}$, increases the anisotropic pre-strain value and consequently self-bending curvature observed experimentally. The pre-strain regime that is anisotropic and speed-dependent as given in table 4 may serve as benchmark data to 4D FDM printing in design, analysis and fabrication.

Next, the developed FE solution is applied to replicate experimental results on triple SMP by 4D printing as presented in figure 7 . In this regard, the pre-strain regime computed from the previous simulation given in table 4 for different printing speeds is employed to perform the present simulation. Three printed beams with prescribed pre-stain regime are programmed in low temperature of $23{ }^{\circ} \mathrm{C}$ by applying bending moment at their edge ends. They are loaded until two end edges meet each other. The cold programming is completed by removing the external moments. The curved beams are then heated up to temperature of $85^{\circ} \mathrm{C}$ and finally cooled down to $23{ }^{\circ} \mathrm{C}$. The configuration of the printed structures before and after cold programming process and also after heating-cooling process is illustrated in figure 10. This is counterpart of figure 7 from simulations. The geometric parameters of the elements after loading, unloading and heating-cooling are also computed and reported in table 2 . The preliminary conclusion drawn from table 2, figures 7 and 10 is that the FE analysis coupled the SMP constitutive model is in an excellent agreement with experiments in terms of configuration of beams during thermo-mechanical loadings along cold programming and cooling-heating processes. The good qualitative and quantitative correlation also verifies 
(a)

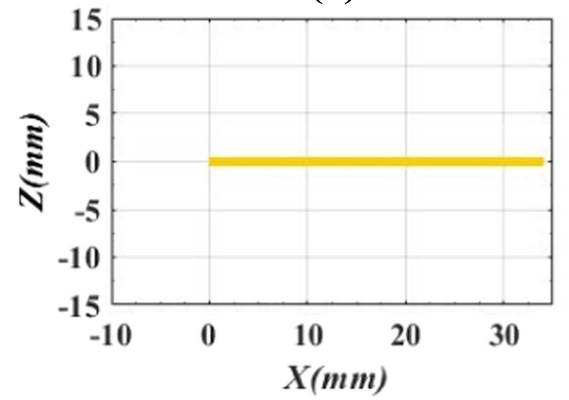

(b)

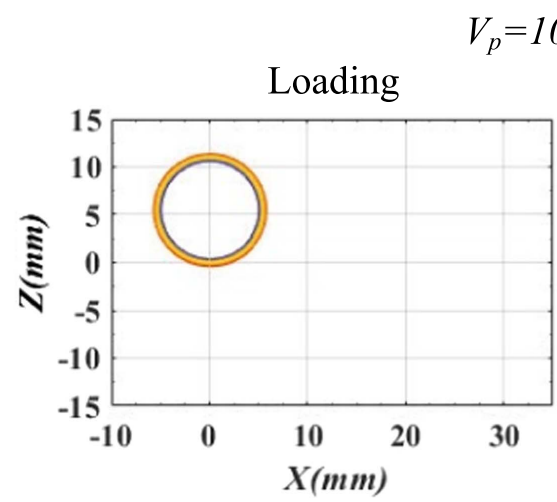

$30,40 \mathrm{mms}^{-1}$

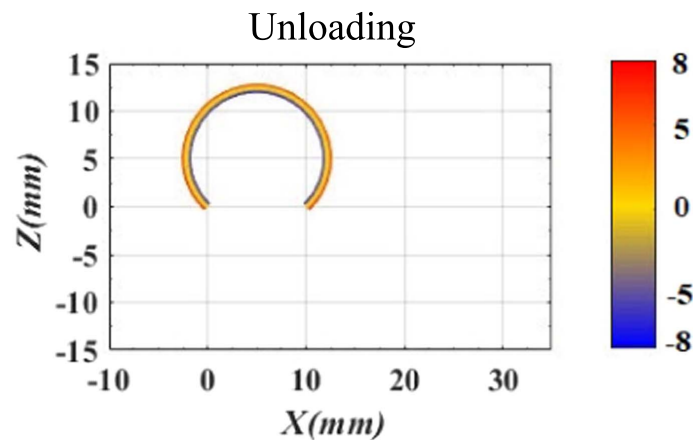

(c)
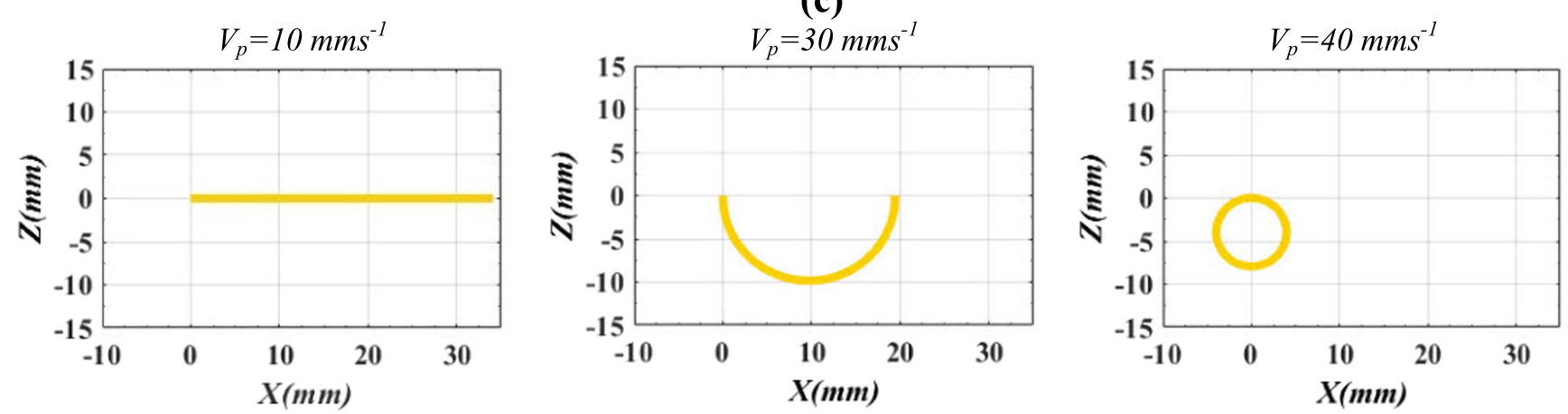

Figure 10. Simulation of triple SMPs presented in figure 7. (color bar shows normal plastic strain (\%) along the circumferential direction)

accuracy and reliability of the pre-strain regimes predicted by dual SME for triple plasticity-based SME. This self-foldable primitive can serve as $1 \mathrm{D}$ standalone stents or mechanical grippers or elements of $3 \mathrm{D}$ stents/grippers. They can be programmed and printed in the 1D beam shape and then placed inside a catheter/tube. Putting the printed beams inside the catheter/tube acts like a cold programming process. They are then delivered to a desired location for deployment purpose. Once the beams with negative curvature are pushed out, they may be heated by means of blood temperature and reveal self-bending feature. The beams printed slowly without any inelastic pre-strain becomes straight releasing plastic strains. They may serve as a supporting function for opening a closed artery and keep blood flowing normally. On the other hand, the beams printed faster enough to induce inelastic pre-strain may self-bend and get positive curvatures. In this case, they may act like a gripper and encompasses any object such as a blood clot for treating stroke. In this respect, the printing speed can be considered as a controllable parameter to customize beam curvature.

Another example is dedicated to demonstrate potential applications of cold programming for self-shrinking/tightening staples. They have become a standard surgical tool for minimally invasive surgery [25]. The feasibility of selfshrinking/tightening staples is demonstrated experimentally and numerically in figure 11. This device consists of one $16 \mathrm{~mm}$ shoulder with two $16 \mathrm{~mm}$ legs filleted by $3 \mathrm{~mm}$ radius as illustrated in figure 11(a). The width and thickness are also $1.6 \mathrm{~mm}$. The printing speed is set as low as $10 \mathrm{~mm} \mathrm{~s}^{-1}$ to prevent inducing any pre-strain. The staple needs a cold programming at $23{ }^{\circ} \mathrm{C}$ and activation by heating up to $85^{\circ} \mathrm{C}$ as illustrated in figures 11(b) and (c). First, horizontal forces are applied to the end of staple legs at temperature of $23{ }^{\circ} \mathrm{C}$ to get maximum opening of $24 \mathrm{~mm}$. The maximum required load is computed as $1.78 \mathrm{~N}$ by the FE analysis. The legs are then unloaded to finally become vertical at the end of unloading stage as shown in figure 11(b). The U-shaped staple 


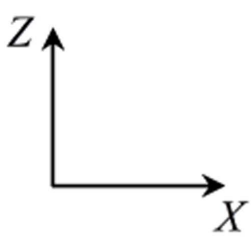

(a)

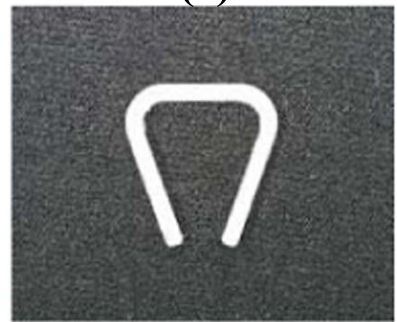

(b)

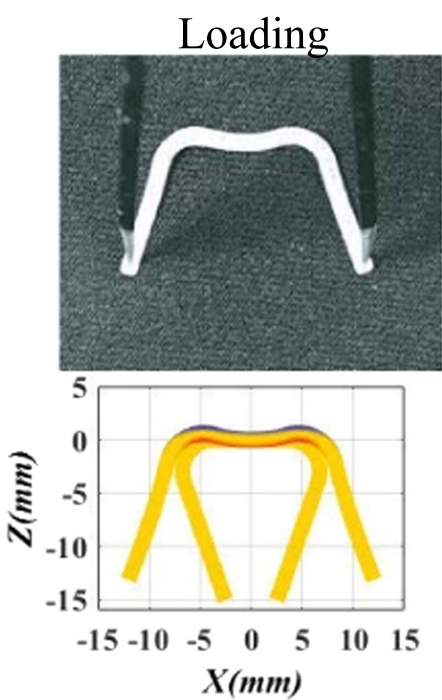

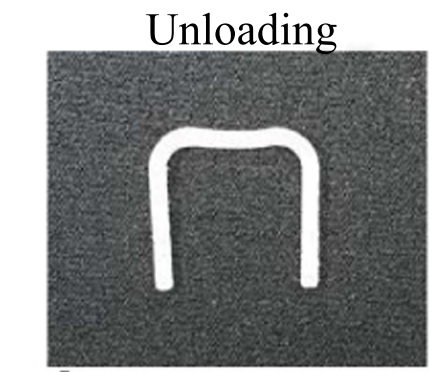

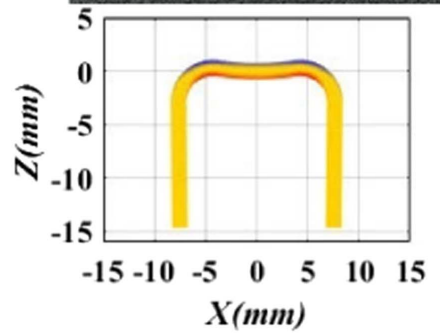

(c)

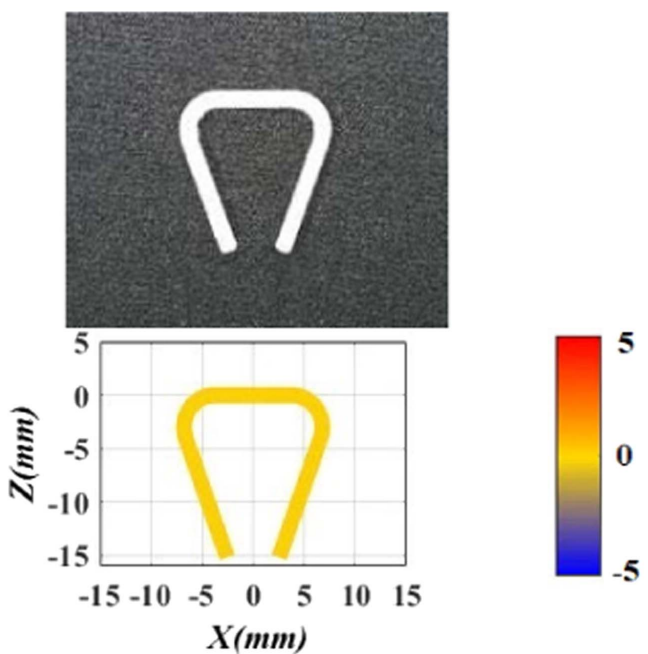

Figure 11. An SMP staple with self-shrinking/tightening feature: its configuration after printing (a), cold programming (b) and heatingcooling (c) processes. (color bar shows normal plastic strain (\%) along the length direction)

can be fired into tissue through small holes. Once it is heated by tissue temperature, it mechanically self-shrinks and recovers back to the original printed shape and would be able to provide gentle force to close gaps like wounds, see figure 11(c). Finally, the results presented in figure 11 reveal high accuracy of the material/structural model in simulating cool programming, plastic deformation, wave-shaped shoulder and shape recovery.

\section{Conclusion}

The present research was dedicated to explore triple SMPs by 4D printing technology and shape adaptive structures with selfbending feature. The concept was on the basis of arranging hotcold programming with FDM printing technology to engineer triple SMPs. Experiments revealed that the printed SMP has elasto-plastic response at low temperatures while it behaves hyper-elastically at high temperatures in the large deformation regime. The feasibility of fabricating and programming SMPs with dual and triple SME and self-bending feature was demonstrated experimentally. To enhance design capabilities of dual/triple SMP by 4D printing, a phenomenological constitutive model was developed incorporating crucial elements including SMP phase transformation, hyper-elasticity, elasto-plasticity and hot-cold programming in framework of large deformation regime. It was then coupled with the FE formulation to simulate 4D printed dual/triple SMP structures through an elastic-predictor plastic-corrector return map algorithm with an excellent accuracy. Experimental and numerical results demonstrated the potential applications of dual/triple SMPs in mechanical and biomedical engineering devices like self-bending gripers/stents and self-shrinking/tightening staples. Due to the absence of similar concept, phenomenological model and results in the specialized literature, this paper is likely to pave the way for designing triple shape adaptive structures by 4D FDM printing technology.

\section{Acknowledgments}

The work described in this paper was supported by the Research Grants Council of the Hong Kong Special 
Administrative Region, China (Project No. CUHK 14202016) and The Chinese University of Hong Kong (Project ID: 3132823).

\section{ORCID iDs}

W H Liao (10) https://orcid.org/0000-0001-7221-5906

\section{References}

[1] Meng H and Li G 2013 A review of stimuli-responsive shape memory polymer composites Polymer 54 2199-221

[2] Liu Y, Du H, Liu L and Leng J 2014 Shape memory polymers and their composites in aerospace applications: a review Smart Mater. Struct. 23023001

[3] Wang K, Strandman S and Zhu X X 2017 A mini review: shape memory polymers for biomedical applications Frontiers Chem. Sci. Eng. 11 143-53

[4] Liu Y, Gall K, Dunn M L, Greenberg A R and Diani J 2006 Thermomechanics of shape memory polymers: uniaxial experiments and constitutive modeling Int. J. Plast. 22 279-313

[5] Park H, Harrison P, Guo Z, Lee M G and Yu W R 2016 Three dimensional constitutive model for shape memory polymers using multiplicative decomposition of the deformation gradient and shape memory strains Mech. Mater. 93 43-62

[6] Li G and Wang A 2016 Cold, warm, and hot programming of shape memory polymers J. Poly. Sci. B 54 1319-39

[7] Momeni F, Hassani S M M, Liu N X and Ni J 2017 A review of 4D printing Mater. Des. 122 42-79

[8] Bodaghi M, Damanpack A R and Liao W H 2016 Selfexpanding/shrinking structures by 4D printing Smart Mater. Struct. 25105034

[9] Kwok T H, Wang C C L, Deng D, Zhang Y and Chen Y 2015 4D printing for freeform surfaces: design optimization of origami and kirigami structures Trans. ASME, J. Mech. Des. 137111712

[10] Yang Y, Chen Y, Wei Y and Li Y 2016 3D printing of shape memory polymer for functional part fabrication Int. J. Adv. Manuf. Technol. 84 2079-95
[11] Hu G F, Damanpack A R, Bodaghi M and Liao W H 2017 Increasing dimension of structures by $4 \mathrm{D}$ printing shape memory polymers via fused deposition modeling Smart Mater. Struct. 26125023

[12] Mao Y, Ding Z, Yuan C, Ai S, Isakov M, Wu J, Wang T, Dunn M L and Qi H J 2016 3D printed reversible shape changing components with stimuli responsive materials Sci. Rep. 624761

[13] Ding Z, Yuan C, Peng X, Wang T, Qi H J and Dunn M L 2017 Direct $4 \mathrm{D}$ printing via active composite materials $S c i . A d v .3$ e1602890

[14] López-Valdeolivas M, Liu D, Broer D J and Sánchez-Somolinos C 2017 4D printed actuators with softrobotic functions Macromol. Rapid Commun. 391700710

[15] Bodaghi M, Damanpack A R and Liao W H 2017 Adaptive metamaterials by functionally graded 4D printing Mater. Des. 135 26-36

[16] Sun L and Huang W M 2010 Mechanisms of the multi-shape memory effect and temperature memory effect in shape memory polymers Soft Matter 6 4403-6

[17] Lu H, Yu K, Huang W M and Leng J 2016 On the Takayanagi principle for the shape memory effect and thermomechanical behaviors in polymers with multi-phases Smart Mater. Struct. 25125001

[18] Lu H, Wang X, Yu K, Huang W M, Yao Y and Leng J 2017 A phenomenological formulation for shape/temperature memory effect in amorphous polymers with multi stress components Smart Mater. Struct. 26095011

[19] Bai Y, Zhang X, Wang Q and Wang T 2014 A tough shape memory polymer with triple-shape memory and two-way shape memory properties J. Mater. Chem. A 2 4771-8

[20] ASTM D638-10 2010 Standard Test Method for Tensile Properties of Plastics pp 1-16

[21] Mooney M 1940 A theory of large elastic deformation J. Appl. Phys. 11582

[22] Truesdell C and Noll W 1965 The Non-Linear Field Theories of Mechanics (Berlin: Springer)

[23] Simo J C and Hughes T J R 1998 Computational Inelasticity (New York: Springer)

[24] Reddy J N 2004 An Introduction to Nonlinear Finite Element Analysis (New York: Oxford University Press)

[25] Huang W M, Song C L, Fu Y Q, Wang C C, Zhao Y, Purnawali H, Lu H B, Tang C, Ding Z and Zhang J L 2013 Shaping tissue with shape memory materials Adv. Drug Deliv. Rev. 65 515-35 